\title{
Effects of Geometrical Clearances, Supports Friction, and Wear Rings on Hydraulic Actuators Bending Behavior
}

\author{
Sergio Baragetti ${ }^{1,2}$ and Francesco Villa ${ }^{1}$ \\ ${ }^{1}$ Department of Management, Information and Production Engineering, University of Bergamo, Viale Marconi 5, \\ 24044 Dalmine, Italy \\ ${ }^{2}$ Centre on Innovation Management and Technology Transfer (GITT), University of Bergamo, Via Salvecchio 19, 24129 Bergamo, Italy
}

Correspondence should be addressed to Sergio Baragetti; sergio.baragetti@unibg.it

Received 28 June 2015; Accepted 10 December 2015

Academic Editor: Jose Merodio

Copyright (c) 2016 S. Baragetti and F. Villa. This is an open access article distributed under the Creative Commons Attribution License, which permits unrestricted use, distribution, and reproduction in any medium, provided the original work is properly cited.

Hydraulic actuators are commonly adopted in machines and structures to provide translating forces with significant magnitudes. Although their application dates back to the industrial revolution, their bending behavior under compression is typically addressed by simple Euler's instability analysis on the rod, neglecting effects such as the cylinder inertia and stiffness, the presence of contact elements in the cylinder-rod junction and on the piston, geometrical misalignments and imperfections, and friction moments at the support. Such simplifications lead to unjustified reduced critical load calculations on the component. In the present paper, a complete mathematical formulation, which accounts for such effects, is presented and validated against experimental data. A numerical sensitivity analysis is conducted, to assess the contributions of initial rectilinear imperfections, wear rings stiffness and dimension, and supports friction on the actuator's limit buckling load and bending behavior under compression. Results are presented, including the effect of the cited parameters on the buckling load, providing a reliable tool for the mechanical designer. In particular, an optimum position for the wear ring distance is found. Moreover, increased wear ring stiffness and reduced imperfections increase the buckling load and reduce the bending stresses before the critical load.

\section{Introduction}

Hydraulic actuators have been widely adopted to apply and multiply translating forces in mechanical systems. Their usage can be traced back at least to the First Industrial Revolution, with the patent of Joseph Bramah in 1795, related to the design of a hydrostatic press based on Blaise Pascal concept $[1,2]$. In modern times, hydraulic actuators are adopted in several applications in the mechanical and civil engineering fields. In particular, their usage is natural whenever a translating linear load of high magnitude is required, due to the elevated hydraulic pressure that can be applied in the cylinder. Despite the wide application fields and the long history of these components, few researchers have addressed the behavior of hydraulic actuators under compression loads.

The bending behavior as well as buckling effect of these devices was considered at first starting from the second half of the XX century, their instability being analyzed by conservative Euler's method. The first models analyzed the bending behavior under compression by considering a single beam instability [3-8]. In such cases, two strategies to model the instability behavior were adopted: a single beam with rod inertial properties and a length equal to the whole actuator [3], or a two-beam structure, with rod and hollow cylinder section definitions $[4,5]$. The results found in classic literature, related to the definition of the actuator critical buckling load, have been demonstrated to be overconservative, as in the single-beam Euler model [3], or overestimating, as the instability model with infinite stiffness hollow cylinder [4]. The limitation of such classical models is the lack of correlation to actual buckling limit load found on working examples, leading often to component oversizing in the best situation, or to unreliable buckling load values, higher than the experimental ones. Besides, the stresses induced by the bending behavior under compression loads were not matching experimental values. 
Moreover, these classical models, as will be seen in their detailed analysis in Section 2.1.1, do not include several effects, which may severely affect the buckling behavior of hydraulic actuators. Indeed, significant factors, such as the operating pressure of the component, its own weight, the actual realization of the cylinder-rod joint at the cylinder gland, and the presence of nonideal, friction pin links have not been considered in these theoretical models.

Further research was hence carried out on the influence of operating parameters and other factors on the actuators buckling. In particular, Flügge [6] and Hoblit [7] included in the model the contribution of the actuator pressure during the compressive load. The effect of the internal pressure resulted as negligible, in terms of its influence on the buckling load value, so that the approximation of considering the cylinder as a hollow component is substantially correct.

Moreover, the cylinder-rod contact in the gland is usually realized by a couple of wear rings, which are the physical supports of the rod. Wear rings can be mounted also on the piston, between the piston outer surface and the cylinder inner surface. Wear rings are adopted to guide the piston and the rod on a reduced wear surface, avoiding metal-tometal contact, reducing friction, and improving the sealing performance, by reducing oil leakage between the components. The presence of wear rings has a nonnegligible impact on the system bending stiffness, and the calculation of its contribution is vital for the determination of the wear rings effects on the bending behavior of the actuator. Theoretical models presenting a welded connection between the rod and the cylinder are obviously not suitable for this kind of analysis.

Another effect of the presence of the wear rings, combined with tolerances coming from the actuator components production process, is the presence of initial rectilinear imperfections. The geometrical clearances found on the practical realization of the actuator lead indeed to an initial transversal displacement, affecting the maximum buckling load at which the actuator can resist.

Finally, the theoretical models present an ideal pin constraint on the actuator ends. However, in practical situations, such assumption is not always negligible. Material pin connections can indeed lead to significant influences on the stress state of the actuator under compression and bending. Even if supported by ball bearings, a certain amount of bending moment is present on the actuator constraints and must be taken into account when evaluating the overall critical load and the applied stress that the actuator can withstand.

The first account of geometrical imperfection effects on actuators was reported by Seshasai et al. [8], where the initial deformation angle due to misalignment between the cylinder and the rod is taken into account. Further investigation on this aspect was carried out by Baragetti and Terranova $[9,10]$. In [9], a first modification of the two-beam model presented by Timoshenko and Gere [5] is proposed. The presence of a rectilinear initial imperfection, due to geometrical clearances, is inserted in the mathematical model. Besides, the effect of the stiffness of two wear rings mounted on the rod is added to the formulation, by means of a bending spring, along with friction moments on the pin supports.
The results of the numerical model are validated against experimental data, resulting in a more accurate critical load prediction with respect to [3], yet with a sufficient safety factor if compared to $[4,5]$. In [10], a more complex rodcylinder interaction is considered, by modeling a system with mounted wear rings not only at the gland-rod interaction, but also between the piston at the rod's end and the external cylinder. The hyperstatic problem associated with this layout is solved, and a bending stiffness equivalent to the combined joint is presented. Again, experimental values are used to validate the model, presenting good agreement with the model results.

The models presented in [8-10] have been recently adopted by Gamez-Montero et al. [11, 12], which inserted the contribution of the actuator own weight and the effect of the cylinder's internal pressure on the buckling load and bending behavior. The model presented in [10] is here modified considering a partial detachment of the wear rings from the internal surfaces of the cylinder and of the gland. In [11], the effects of misalignment are addressed, substantially confirming the validity of the theoretical approach presented in $[9,10]$. The consideration by Flügge [6], that the internal pressure resulted in negligible effects, is also confirmed in [11]. Misalignment produces indeed a remarkable influence in the final buckling limit load, if compared to internal pressure. The actuator own weight contribution seems also to be negligible, providing only a slight modification of the limit load. Besides, including the actuator own weight might be questionable, whenever the actuator is not mounted in a horizontal position, as considered in [11]. In [12], numerical and experimental pin interactions, which produce moderateto-severe friction conditions, are investigated. The results of [12] are again concurring with $[9,10]$. The severe influence of the friction effect on pin supports regarding the bending behavior and the final buckling load is hence to be taken into account and validated against the factual constraint conditions. To conclude the description of the current state of the art, a mention of the ISO standard 13725 [13] is needed. The ISO standard proposes indeed a method to calculate the cylinders buckling load, but its partial description of the problem has been highlighted by Gamez-Montero et al. in [11, 12]. In particular, ISO standard [13] considers the possibility of load eccentricity and different boundary conditions, as well as the structural contribution of the rod. However, the presence of angular imperfections, of friction on the supports, and wear ring influence are not considered, according to [11]. Moreover, the current version of the standard [13] is retired, and a new standard is under development at the moment of print of the present work.

In order to finalize the findings of the actual state of the art, as just presented in the previous paragraph [8-12], the different effects identified in literature works must be summed up in a single numerical model. A study of the influence of such parameters is mandatory, to provide a unified tool for the mechanical designers. Considerations on the effects of the design parameters on the buckling load and bending stresses must be also investigated with sensitivity analyses. The aim of the present paper is hence to analyze the effect of the actuator design parameters and 
material imperfections on the bending behavior of hydraulic actuators under compression loads. The theoretical model, which aims to give insight on the actuator's design parameters and practical application phenomena, should include all the actual effects, which may affect such behavior. The material actuator realization presents indeed several factors, which are not negligible in calculating the actuator buckling load and its bending behavior under compression, according to [9-12]. The contribution of wear rings on flexural stiffness will be analyzed according to $[9,10]$, considering both wear ring couples mounted on the rod-gland connection and rings mounted on the piston-cylinder contact. In Section 2, a review of the classical theoretical methods [3-5] and more advanced methods, which include geometrical imperfections and wear ring stiffness influences $[9,10]$, is presented. From the theoretical models, a complete formulation is chosen and implemented in a commercial code. FE models are produced to assess with extreme precision the transversal stiffness of the wear ring components, depending on the material. The overall results are validated against experimental data. The numerical model thus obtained is adopted to study the influence of the presented imperfections and practical realizations on the bending behavior of the actuator under compression. The proposed method is suitable to analyze most of the mechanical effects, which are present due to components adopted in the modern configuration of hydraulic actuators. Examples of this complexity may be found in the geometry well described in [14], concerning a state-of-the-art device employed in excavator arms. The effects of wear rings and other mechanical interfaces between the piston and the rod can be taken into account by means of the mixed numerical model and FE approach proposed in this work.

\section{Materials and Methods}

\subsection{Numerical Models}

2.1.1. Classical Models from Instability Theory. The most conservative, classic Euler's critical load for columns [3, 4] applied to a simplified rod model leads to

$$
P=\frac{\pi^{2} E J_{s}}{l^{2}}
$$

with $E$ Young's modulus and $J_{s}$ inertial momentum related to the rod section, and $l$ is the unsupported length of column, to be assumed as the whole length of the actuator if the extremes are pin supported. Expression (1) leads to the most conservative buckling load value, and it is considered valid in the elastic field of the material. Quite obviously, (1) completely neglects the effects of geometrical clearance, initial rectilinear effects, stiffness effects due to wear rings in the junction, and friction effects. Moreover, the influence of the cylinder inertial properties on the system stiffness is not considered at all. In [4], the hollow cylinder inertia is modeled as infinite, leading to the transcendental equation:

$$
b \sqrt{\frac{P}{E J_{s}}}+\operatorname{tg}\left(a \sqrt{\frac{P}{E J_{s}}}\right)=0 .
$$

$P$ is the compression load, $E J_{s}$ is related to the rod's section properties, $a$ is the length of the extended portion of the rod outside the cylinder, and $b$ the length of the actuator's cylinder, as reported in Figure 1. If compared to Euler's critical column load presented in (1), the critical load obtained from (2) produces overestimating load values, individuating the upper extreme of the actual limit load of the actuator, while (1) represents the lower, overconservative extreme.

A more sophisticated buckling model, which takes into account the actual moment of inertia of the cylinder, is proposed by Timoshenko and Gere [5] and compared to Euler's model in Figure 1. The elastic deflection equation includes the contribution of both the cylinder and the rod inertial properties. The value of the rod transversal deflection $y_{s}$, function of the axial coordinate $x$, as indicated in Figure 1, is found according to (3). The rod extended shaft length is indicated with $a$ and its inertial momentum with $J_{s}$ :

$$
y_{s}^{\prime \prime}+\frac{P}{E J_{s}} y_{s}=0, \quad 0 \leq x \leq a
$$

Equation (3) presents the general integral:

$$
\begin{aligned}
y_{s}(x) & =A_{s} \sin \left(\alpha_{s} x\right)+B_{s} \cos \left(\alpha_{s} x\right), \\
\alpha_{s} & =\sqrt{\frac{P}{E J_{s}}} .
\end{aligned}
$$

Equation (6) for the elastic deflection can be written also for the cylinder, given its length $b$ and its inertia $J_{c}$. The cylinder's transversal deflection is indicated as $y_{c}$, and it is a function of the same axial coordinate $x$. The general integral (7)-(8) is reported as well:

$$
\begin{aligned}
y_{c}^{\prime \prime}+\frac{P}{E J_{c}} y_{c} & =0, \quad a \leq x \leq a+b, \\
y_{c}(x) & =A_{c} \sin \left(\alpha_{c} x\right)+B_{c} \cos \left(\alpha_{c} x\right), \\
\alpha_{c} & =\sqrt{\frac{P}{E J_{c}}} .
\end{aligned}
$$

The boundary conditions at the pinned ends of the actuator are set as follows:

$$
\begin{array}{r}
y_{s}(0)=0, \\
y_{c}(a+b)=0,
\end{array}
$$

while the junction between the cylinder and the rod, represented in this model as if it were a weld, leads to the following conditions on displacement and rotation:

$$
\begin{aligned}
& y_{c}(a)=y_{s}(a), \\
& y_{c}^{\prime}(a)=y_{s}^{\prime}(a) .
\end{aligned}
$$




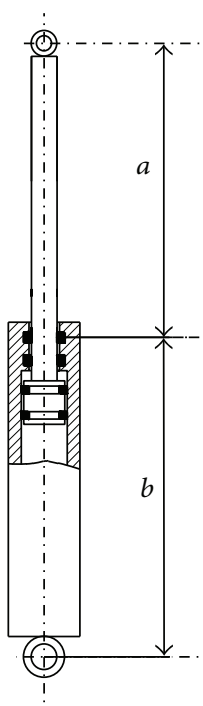

(a)

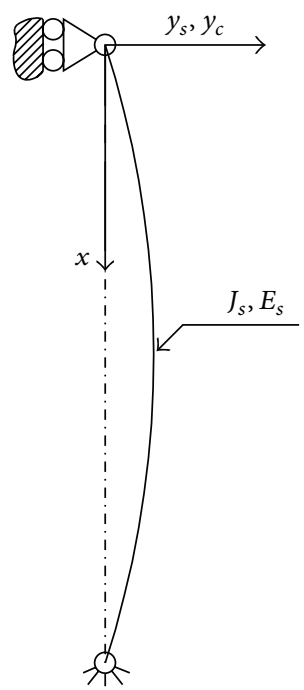

(b)

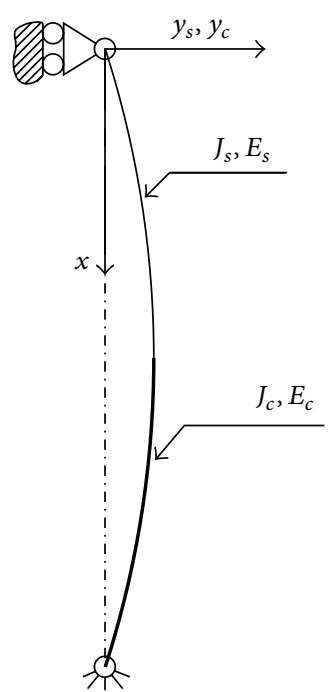

(c)

FIGURE 1: Classic Euler's instability buckling models and geometries: (a) drawing of the actuator; (b) Euler's single beam model; (c) Timoshenko rod and cylinder model.

Condition (9a) leads to $B_{s}=0$, while if adding conditions (9c)-(9d), a three-equation system in the unknowns $A_{s}, A_{c}$, and $B_{c}$ can be written, as shown in

$$
\begin{aligned}
& {\left[\begin{array}{ccc}
0 & \sin \left(\alpha_{c}(a+b)\right) & \cos \left(\alpha_{c}(a+b)\right) \\
\sin \left(\alpha_{s} a\right) & -\sin \left(\alpha_{c} a\right) & -\cos \left(\alpha_{c} a\right) \\
\alpha_{s} \cos \left(\alpha_{s} a\right) & -\alpha_{c} \cos \left(\alpha_{c} a\right) & \alpha_{c} \cos \left(\alpha_{c} a\right)
\end{array}\right]\left\{\begin{array}{l}
A_{s} \\
A_{c} \\
B_{c}
\end{array}\right\}} \\
& \quad=\left\{\begin{array}{l}
0 \\
0 \\
0
\end{array}\right\} .
\end{aligned}
$$

The solution with nonnull coefficients is different from zero only if the determinant of the coefficients' matrix is null, hence leading to the transcendental equation

$$
\begin{aligned}
\alpha_{s}[ & \left.\tan \left(\alpha_{c} a\right)-\tan \left(\alpha_{c}(a+b)\right)\right] \\
& -\alpha_{c} \tan \left(\alpha_{s} a\right)\left[1+\tan \left(\alpha_{c} a\right) \tan \left(\alpha_{c}(a+b)\right)\right] \\
= & 0 .
\end{aligned}
$$

The lower solution of the transcendental equation (11) leads to the buckling load of the actuator, considering the inertial properties of both the cylinder and the rod. Nonideal effects such as rectilinear imperfections, friction moments at the pin supports, and stiffness of the rod-cylinder connection are however still neglected.

The model presented from Timoshenko and Gere [5], leading to the critical load from the solution of (10), still represents an ideal formulation of the actuator problem. As mentioned in the introduction, the aim of the present work is to assess the effects of rectilinear imperfections, due to initial geometrical clearances resulting in an initial transversal displacement, the influence of friction moments

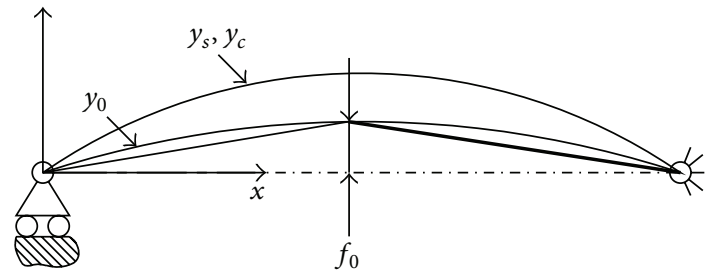

FIgURE 2: Rectilinear deformation of initial configuration.

on the pin supports, and the contribution in terms of bending stiffness due to the adoption of wear rings. In the following subsections, modifications to model (3)-(11) will be presented, taking into account these contributions.

2.1.2. Rectilinear Imperfection. Initial geometrical clearances, caused by production tolerances on cylinder, rod, piston, and gland, lead to an inevitable rectilinear imperfection, which must be taken into account when calculating the cylinder buckling load. The presence of wear rings, each one with its tolerances, as well as their adaptation in the application site introduces further contribution to rectilinear imperfection. To take into account this phenomenon, the initial actuator deflection $y_{0}$ must be considered. The rod-cylinder deflection is schematized as in Figure 2, by imposing a sinusoidal deformation of the form (12), as reported in [10]:

$$
\begin{aligned}
y_{0}(x) & =m \sin (\beta x), \\
\beta & =\frac{\pi}{a+b}, \\
m & =\frac{f_{0}}{\sin (\pi /(1+b / a))} .
\end{aligned}
$$


In (12), $y_{0}$ is the initial transversal displacement along the axis coordinate $x$, as depicted in Figure 2, so that the maximum deflection $f_{0}$ is found at the cylinder-rod intersection.

The rod elastic displacement behavior can be hence modeled as

$$
P y_{s}=-E J_{s}\left(y_{s}^{\prime \prime}-y_{0 s}^{\prime \prime}\right), \quad 0 \leq x \leq a
$$

which can be rewritten as

$$
\alpha_{s}^{2} y_{s}=-y_{s}^{\prime \prime}+y_{0 s}^{\prime \prime}
$$

thus leading to (17) for the rod, where the initial displacement is seen as an external forcing:

$$
y_{s}^{\prime \prime}+\alpha_{s}^{2} y_{s}=-m \beta^{2} \sin (\beta x) .
$$

Equation (17) solution can be obtained as

$$
\begin{array}{r}
y_{s}=A_{s} \sin \left(\alpha_{s} x\right)+B_{s} \cos \left(\alpha_{s} x\right)+\frac{m \beta^{2}}{\beta^{2}-\alpha_{s}^{2}} \sin (\beta x), \\
0 \leq x \leq a .
\end{array}
$$

For the cylinder, a similar equation can be obtained from the same procedure:

$$
\begin{array}{r}
y_{c}=A_{c} \sin \left(\alpha_{c} x\right)+B_{c} \cos \left(\alpha_{c} x\right)+\frac{m \beta^{2}}{\beta^{2}-\alpha_{c}{ }^{2}} \sin (\beta x), \\
a \leq x \leq a+b .
\end{array}
$$

Equations (18), (19) can be solved as a system, by imposing the boundary conditions $(9 a)-(9 d)$, being the same of problem (18), (19).

2.1.3. Influence of Friction. In real applications, friction moments at the pin connection can be adopted to simulate actual working conditions. According to the formulation reported in $[9,10]$, the friction moment is included in the forcing of the elastic displacement equation for the rod:

$$
P y_{s}=-E J_{s} y_{s}^{\prime \prime}+M_{r}, \quad 0 \leq x \leq a .
$$

The differential equation (20) presents the following solution for the transversal displacement of the rod:

$$
y_{s}=A_{s} \sin \left(\alpha_{s} x\right)+B_{s} \cos \left(\alpha_{s} x\right)+\frac{M_{s}}{\alpha_{s}^{2}}, \quad 0 \leq x \leq a,
$$

and an analogous equation can be written for the cylinder displacement:

$$
\begin{aligned}
y_{c}=A_{c} \sin \left(\alpha_{c} x\right)+B_{c} \cos \left(\alpha_{c} x\right)+\frac{M_{c}}{\alpha_{c}{ }^{2}}, & \\
& a \leq x \leq a+b .
\end{aligned}
$$

The terms $M_{s}$ and $M_{c}$ are defined after the applied friction moment $M_{r}$ and the inertial properties of the rod and the cylinder:

$$
\begin{aligned}
& M_{s}=\frac{M_{r}}{E_{s} J_{s}}, \\
& M_{c}=\frac{M_{r}}{E_{c} J_{c}} .
\end{aligned}
$$

For a determination of the friction model, considering actuators mounted on steel pins, the expression from the work by Gamez-Montero et al. [12] may be adopted, neglecting the shear reactions and considering the axial load, as

$$
M_{r}=\frac{1}{4} f d_{\text {pin }} \frac{1-\cos \alpha_{0}}{\left(2 \alpha_{0}-\sin \alpha_{0}\right)} \alpha_{0} P
$$

where the pressure load distribution angle $\alpha_{0}$ can be taken as $\pi / 2$ as a first approximation.

If considering actuators mounted on ball-bearings, the friction moment can be considered negligible. However, bearer manufacturers often provide friction models, which may be used to determine the friction moment starting from an applied load. Considering SKF ball-bearings manufacturing indications [15], the friction moment can be estimated as

$$
M_{r}=\frac{1}{2} f_{\mathrm{bb}} P d_{\mathrm{bb}}
$$

where $f_{\mathrm{bb}}=0.0015$ is the ball-bearing friction coefficient, according to [15], and $d_{\mathrm{bb}}$ is the inner support diameter.

2.1.4. Bending Stiffness Contribution. As already discussed in the introduction, the actual realization of the rod-gland sealing and of the piston-cylinder sliding contact includes elastic effects from the wear rings deformation, affecting the global system stiffness. In order to include the elastic deformation of the wear rings in the system, the pistonrod interaction has been modeled by introducing a bending spring in the mathematical model. The spring must reproduce the effect on the system stiffness generated by the presence of the wear rings. Two kinds of wear rings layout are currently reproduced in hydraulic actuators: wear ring sealing between the rod and the gland, for single actuating cylinders, as depicted in Figure 3, and a combination of wear rings between rod-gland and piston-cylinder surface interactions, as presented in Figure 4, typical for double acting cylinders.

The two proposed layouts can be reduced to a bending spring $K_{f}$, which depends on the wear rings material, dimensioning, and position. The placement of a couple of wear rings at the gland-rod interaction, as in Figure 3, results in an isostatic connection between the piston and the cylinder, as reported in [9]. On the contrary, the presence of additional wear rings between the cylinder and the piston, as reported in Figure 4, leads to a hyperstatic problem for the $K_{f}$ determination, following the approach reported in [10].

In a first stance, the determination of the $K_{f}$ value is proposed for a simple gland-rod junction with two wear rings, as in Figure 3. The mean value of the wear rings deflection, indicated as

$$
\Delta s_{m}=\theta \frac{\delta}{2}
$$

generates a mean pressure:

$$
p_{m}=\frac{\Delta s_{m}}{s} E_{a}=\theta \frac{\delta}{2 s} E_{a},
$$

where $\theta$ is the rod rotation at the interface and $E_{a}$ is Young's modulus of the wear rings. If we distribute the pressure force 


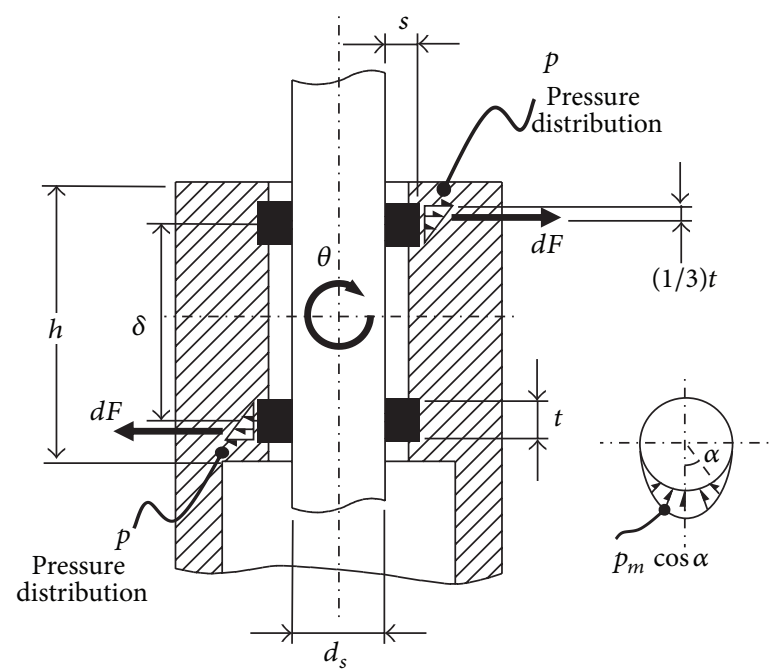

Figure 3: Wear rings between rod and gland, actual realization and theoretical model.

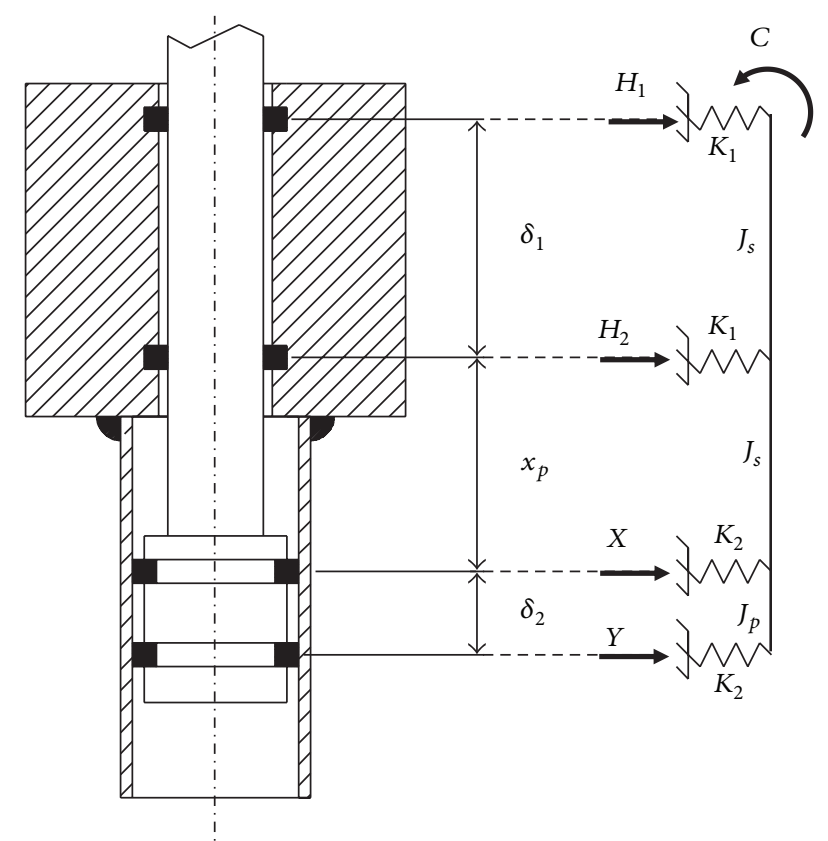

Figure 4: Wear rings between rod and gland and between piston and cylinder, actual realization and theoretical PVW model.

along an elementary area along the circumferential direction $d c=d_{s} / 2 \cdot d \alpha$ we obtain the elementary pressure force:

$$
d F=p_{m} t \frac{d_{s}}{2} d \alpha
$$

As can be seen in Figure 3, if a triangular linear distribution of the pressure on the wear ring internal surface along the $x$-axis is considered, the elementary resultant of the pressure force is applied at $(1 / 3) t$ of the wear ring width. The triangular distribution is coherent with the deformation introduced in the wear ring by a rotation $\theta$, at least as a first approximation. $p_{m}$ is hence the mean pressure associated with the triangular distribution. The elementary moment is hence obtained in (29) as follows:

$$
d M=p_{m} t \frac{d_{s}}{2} d \alpha\left(\delta+\frac{t}{3}\right) .
$$

Along the circumferential direction, the pressure distribution is modeled along a cosine law of the form

$$
p_{m}(\alpha)=\theta \frac{\delta}{2 s} E_{a} \cos \alpha
$$

between $\alpha=-\pi / 2$ and $\alpha=\pi / 2$, as reported in Figure 3, reaching the maximum value of mean pressure $p_{m}$ for $\alpha=0$.

Similarly, if we consider the elementary moment along the circumferential direction,

$$
\begin{aligned}
d M(\alpha) & =2 d F(\alpha) \cos \alpha \frac{(\delta+t / 3)}{2} \\
& =p_{m} t \frac{d_{s}}{2} d \alpha\left(\delta+\frac{t}{3}\right) \cos \alpha \\
& =\theta \frac{\delta}{2 s} E_{a} t \frac{d_{s}}{2}\left(\delta+\frac{t}{3}\right) \cos ^{2} \alpha d \alpha .
\end{aligned}
$$

By integrating the elementary moment between $\alpha=-\pi / 2$ and $\alpha=\pi / 2$,

$$
M=\int_{-\pi / 2}^{\pi / 2} d M(\alpha)=K_{f} \theta
$$

the junction bending stiffness $K_{f}$ can hence be obtained as

$$
K_{f}=\frac{\delta E_{a} \pi t d_{s}}{8 s}\left(\delta+\frac{t}{3}\right) .
$$

For double acting cylinders, the presented model, which results in a bending stiffness of the junction $K_{f}$ as obtained in (33), is valid no more. Indeed, the presence of a piston acting in both ways requires wear rings to be mounted also between the cylinder and the piston external surface, as in Figure 4.

The elastic system resulting from this layout differs significantly from the effects of a junction as presented in Figure 3. The interface indeed presents two wear rings supporting the rod, acting as parallel springs, as well as two wear rings between the piston and the cylinder, with the same behavior. To calculate the bending stiffness of the resulting hyperstatic system, the principle of virtual work (PVW) approach can be followed.

Indeed, if a virtual moment $C$, which can be considered as unitary, is applied at the end of the interface, as in Figure 4, the correspondent rotation $\theta$ can be calculated by solving the system (34), and the bending stiffness obtained from $K_{f}=$ $C / \theta$ :

$$
\left[\begin{array}{ll}
C_{11} & C_{12} \\
C_{21} & C_{22}
\end{array}\right]\left\{\begin{array}{l}
X \\
Y
\end{array}\right\}=\left\{\begin{array}{l}
T_{1} \\
T_{2}
\end{array}\right\} C
$$


The coefficients of system (34) can be calculated as

$$
\begin{aligned}
& C_{11} \\
& =-\frac{1}{K_{2}}-\frac{\left(\delta_{1}+x_{p}\right)^{2}}{\delta_{1}{ }^{2} K_{1}}-\frac{x_{p}{ }^{2}}{\delta_{1}{ }^{2} K_{1}}-\frac{x_{p}{ }^{2} \delta_{1}+x_{p}{ }^{3}}{3 E J_{s}} \\
& -\frac{\chi_{s}}{G A_{s}}\left(\frac{x_{p}^{2}}{\delta_{1}}+\delta_{1}\right) \\
& C_{12} \\
& =-\frac{\left(\delta_{1}+\delta_{2}+x_{p}\right)\left(\delta_{1}+x_{p}\right)}{\delta_{1}^{2} K_{1}}-\frac{\left(\delta_{2}+x_{p}\right) x_{p}}{\delta_{1}{ }^{2} K_{1}} \\
& -\frac{1}{E J_{s}}\left[\frac{1}{3} x_{p} \delta_{1}\left(x_{p}+\delta_{2}\right)+\frac{1}{3} x_{p}{ }^{3}+\frac{1}{2} \delta_{2} x_{p}{ }^{2}\right] \\
& -\frac{\chi_{s}}{G A_{s}}\left[\frac{x_{p}\left(x_{p}+\delta_{2}\right)}{\delta_{1}}+x_{p}\right] \text {, } \\
& C_{12}=C_{21} \text {, } \\
& \mathrm{C}_{22} \\
& =-\frac{\left(\delta_{2}+x_{p}\right)^{2}}{\delta_{1}^{2} K_{1}}-\frac{\left(\delta_{1}+\delta_{2}+x_{p}\right)^{2}}{\delta_{1}^{2} K_{1}}-\frac{1}{K_{2}} \\
& -\frac{1}{E J_{s}}\left[\frac{1}{3} \delta_{1}\left(x_{p}+\delta_{2}\right)^{2}+\frac{1}{3} x_{p}{ }^{3}+\delta_{2} x_{p}{ }^{2}+\delta_{2}{ }^{2} x_{p}\right] \\
& -\frac{1}{E J_{p}}\left(\frac{1}{3} \delta_{2}^{3}\right)-\frac{\chi_{s}}{G A_{s}}\left[\frac{\left(x_{p}+\delta_{2}\right)^{2}}{\delta_{1}}+x_{p}\right] \\
& -\frac{\chi_{p}}{G A_{p}} \delta_{2} \\
& T_{1}=\frac{\delta_{1}+2 x_{p}}{\delta_{1}^{2} K_{1}}-\frac{1}{E J_{s}} \frac{1}{6} \delta_{1} x_{p}+\frac{\chi_{s}}{G A_{s}} \frac{x_{p}}{\delta_{1}}, \\
& T_{2} \\
& \begin{aligned}
= & \frac{\left(\delta_{2}+x_{p}\right)}{\delta_{1}^{2} K_{1}}+\frac{\left(\delta_{1}+\delta_{2}+x_{p}\right)}{\delta_{1}^{2} K_{1}}-\frac{\left(\delta_{2}+x_{p}\right) \delta_{1}}{6 E J_{s}} \\
& +\frac{\chi_{s}}{G A_{s}} \frac{\left(\delta_{2}+x_{p}\right)}{\delta_{1}} .
\end{aligned}
\end{aligned}
$$

Once the unknown reactions $X$ and $Y$ are obtained from the system (34), the rotation $\theta$ can be obtained from

$$
\begin{aligned}
& \theta=T_{1} X+T_{2} Y+Z C, \\
& Z=\frac{2}{\delta_{1}^{2} K_{1}}+\frac{\delta_{1}}{3 E J_{s}}+\frac{\chi_{s}}{\delta_{1} G A_{s}} .
\end{aligned}
$$

The bending stiffness of the system can be hence obtained from

$$
K_{f}=\frac{C}{\theta}
$$

remembering that $C$ is an arbitrary virtual moment value, which can be set as unitary.

In order to obtain the linear stiffness of $K_{1}$ and $K_{2}$ related to the wear rings, a finite element (FE) model has been built, using the Abaqus software, specifically for the numerical method presented in this work. The rod has been modeled as a rigid body surface, while the wear ring has been discretized with solid linear hexahedral elastic elements (C3D8R). The mesh size was of $0.1 \mathrm{~mm}$ in the region where the relative displacement $\Delta x$ was calculated, and of $0.3 \mathrm{~mm}$ in the other regions, to improve the calculation time. The ring material was modeled after actual wear ring properties $[9,10]$, with a linear elastic, isotropic, and homogenous constitutive law. The rod surface was constrained in all its degrees of freedom (DOFs), except along the $X$ direction, in which a force $F$ was applied, as can be seen in Figure 5(a). The external surface of the wear ring was constrained in the radial direction alone, simulating contact between the ring and the outer supporting surface, while the ring was kept free to expand along the axial direction. To reduce the complexity of the model, only half of the geometry was modeled, imposing symmetry on the $X-Y$ plane, as reported in Figure 5(a). A normal contact property was imposed between the ring and the rod surfaces.

The relative displacement between the deformed and undeformed configurations, as seen in Figure 5(c), is calculated as

$$
\Delta x=\Delta_{0}-\Delta .
$$

The linear stiffness of each wear ring is hence calculated from

$$
K=\frac{F}{\Delta x} \text {. }
$$

Once the interface bending stiffness $K_{f}$ has been obtained, from (33) or (37) depending on the system layout, the parameter can be introduced in the elastic displacement theoretical model as presented in Figure 6.

The differential equation ruling the system of Figure 6 is the same as the system without interface stiffness modeling, that is, (3) and (6) for the rod and the cylinder, respectively. The integral solution form is as well the same for both components, as in (4), (7). The difference stands in the boundary conditions of the problem. Indeed, the relative rotation at the interface between rod and cylinder, dependent on the bending stiffness $K_{f}$, modifies the boundary conditions presented in (9d). The rotation between the rod and the cylinder at the interface cannot be considered equal as in (9d), but it must be adapted to guarantee the equality between the moment of the applied axial load $P$ and the elastic moment given by $K_{f}$.

According to the theoretical approach of $[9,10],(9 d)$ is hence substituted by the rotational equilibrium:

$$
P y_{s}(a)=K_{f}\left[y_{s}^{\prime}(a)-y_{c}^{\prime}(a)\right] \text {. }
$$

By substituting (4) and (7), related to rod and cylinder deflections $y_{s}$ and $y_{c}$, respectively, the following equation is obtained:

$$
\begin{aligned}
& P A_{s} \sin \left(\alpha_{s} a\right)=K_{f}\left[A_{s} \cos \left(\alpha_{s} a\right) \alpha_{s}-A_{c} \cos \left(\alpha_{c} a\right) \alpha_{c}\right. \\
& \left.+B_{c} \sin \left(\alpha_{c} a\right) \alpha_{c}\right] .
\end{aligned}
$$




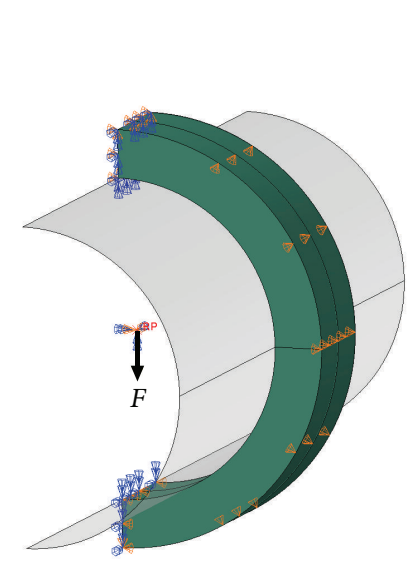

(a)

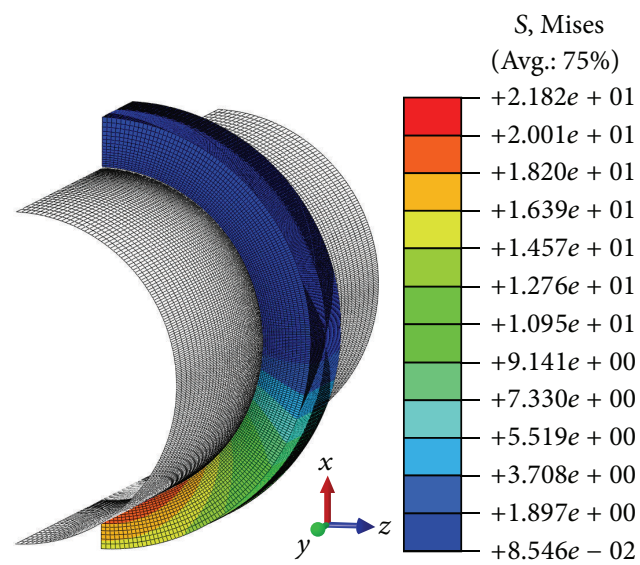

(b)

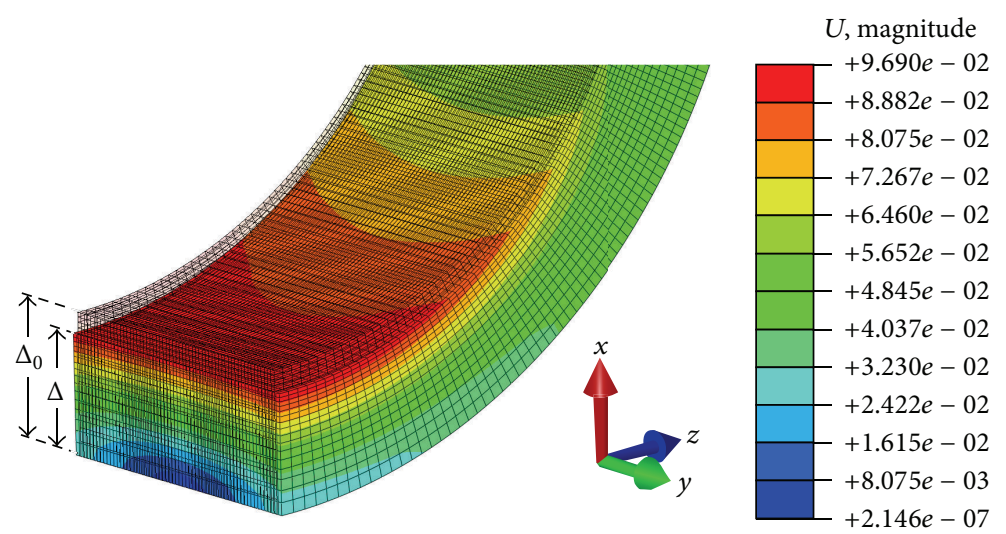

(c)

Figure 5: Finite element model adopted for the determination of the wear rings linear stiffness $K$ : (a) loads and constraints; (b) stress distribution after compression; (c) vertical displacement [mm] after compression, and coordinates for the relative displacement calculation.

Equation (41) substitutes the third line of system (10), resulting in

$$
\left[\begin{array}{ccc}
0 & \sin \left(\alpha_{c}(a+b)\right) & \cos \left(\alpha_{c}(a+b)\right) \\
\sin \left(\alpha_{s} a\right) & -\sin \left(\alpha_{c} a\right) & -\cos \left(\alpha_{c} a\right) \\
P \sin \left(\alpha_{s} a\right)-K_{f}\left[\cos \left(\alpha_{s} a\right)\right] \alpha_{s} & K_{f} \cos \left(\alpha_{c} a\right) \alpha_{c} & -K_{f} \sin \left(\alpha_{c} a\right) \alpha_{c}
\end{array}\right]\left\{\begin{array}{l}
A_{s} \\
A_{c} \\
B_{c}
\end{array}\right\}=\left\{\begin{array}{l}
0 \\
0 \\
0
\end{array}\right\} .
$$

System (42) presents a nonnull solution related to the variable $P$, included in $\alpha_{s}$ and $\alpha_{c}$, only if the determinant of its matrix equals zero, leading to the transcendental equation:

$$
\begin{aligned}
& \left\lfloor P \tan \left(\alpha_{s} a\right)-K_{f} \alpha_{s}\right\rfloor\left[\tan \left(\alpha_{c} a\right)-\tan \left(\alpha_{c}(a+b)\right)\right] \\
& \quad+\alpha_{c} K_{f} \tan \left(\alpha_{s} a\right)\left[1+\tan \left(\alpha_{c} a\right) \tan \left(\alpha_{c}(a+b)\right)\right] \\
& \quad=0 .
\end{aligned}
$$

The solution of (43) with respect to $P$ results in the critical load for an actuator having an interface between rod and cylinder with finite stiffness $K_{f}$.
2.1.5. Combined Model: Bending Stiffness, Friction Moment, and Rectilinear Imperfection. In the study of a current realization of a hydraulic actuator, the effects presented in Sections 2.1.2-2.1.4 are usually found together. To assess their combined impact on the actuator buckling load, the solution of a proper model must be developed. The complete theoretical model, obtained from $[9,10]$ and presented in Figure 7, and the effects of rectilinear imperfections caused by geometrical clearances, of friction moments on the supports, and of a junction of finite bending stiffness are analyzed. 


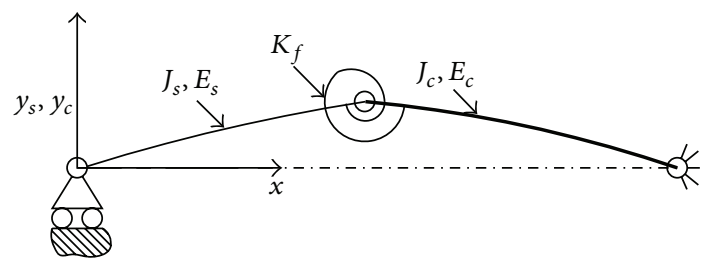

FIgURE 6: Theoretical model for elastic displacement of rod and cylinder, considering the interface bending stiffness $K_{f}$.

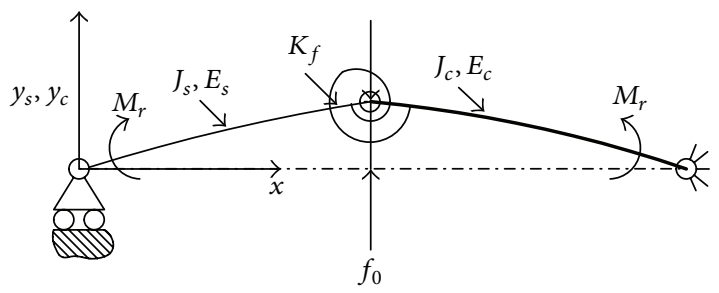

FIgURE 7: Theoretical model of pin friction along with bending stiffness from wear rings between rod and gland and between piston and cylinder, and initial rectilinear imperfection.

Including friction moments and the initial rectilinear imperfection displacement $y_{0}(x)=m \sin (\beta x)$, the equations for the deflection of rod shaft and cylinder are hence

$$
\begin{array}{ll}
y_{s}^{\prime \prime}+\alpha_{s}^{2} y_{s}=-m \beta^{2} \sin (\beta x)+M_{s}, & 0 \leq x \leq a, \\
y_{c}^{\prime \prime}+\alpha_{c}^{2} y_{c}=-m \beta^{2} \sin (\beta x)+M_{c}, & a \leq x \leq a+b .
\end{array}
$$

General integrals of (44) are

$$
\begin{aligned}
y_{s}= & A_{s} \sin \left(\alpha_{s} x\right)+B_{s} \cos \left(\alpha_{s} x\right)+\frac{m \beta^{2}}{\beta^{2}-\alpha_{s}^{2}} \sin (\beta x) \\
& +\frac{M_{s}}{\alpha_{s}^{2}}, \quad 0 \leq x \leq a, \\
y_{c}= & A_{c} \sin \left(\alpha_{c} x\right)+B_{c} \cos \left(\alpha_{c} x\right)+\frac{m \beta^{2}}{\beta^{2}-\alpha_{c}^{2}} \sin (\beta x) \\
& +\frac{M_{c}}{\alpha_{c}^{2}}, \quad a \leq x \leq a+b .
\end{aligned}
$$

Boundary conditions to be applied to system (45), (46) are hence including the interface bending stiffness $K_{f}$ from (40):

$$
\begin{aligned}
y_{s}(0) & =0, \\
y_{c}(a+b) & =0, \\
y_{c}(a) & =y_{s}(a), \\
P y_{s}(a) & =K_{f}\left[y_{s}^{\prime}(a)-y_{c}^{\prime}(a)\right] .
\end{aligned}
$$

Condition (47a) leads to define the $B_{s}$ constant:

$$
B_{s}=-\frac{M_{s}}{\alpha_{s}^{2}}
$$

thus leading to the following reformulation of (45):

$$
\begin{aligned}
y_{s}= & A_{s} \sin \left(\alpha_{s} x\right)-\frac{M_{s}}{\alpha_{s}{ }^{2}} \cos \left(\alpha_{s} x\right) \\
& +\frac{m \beta^{2}}{\beta^{2}-\alpha_{s}{ }^{2}} \sin (\beta x)+\frac{M_{s}}{\alpha_{s}{ }^{2}} .
\end{aligned}
$$

Condition (47b) leads to the following relation:

$$
A_{c} \sin \left[\alpha_{c}(a+b)\right]+B_{c} \cos \left[\alpha_{c}(a+b)\right]+\frac{M_{c}}{\alpha_{c}^{2}}=0
$$

By imposing equal vertical displacements between the rod and the cylinder, as in condition $(47 \mathrm{c})$, another relation can be written for the constants $A_{s}, A_{c}$, and $B_{c}$ :

$$
\begin{aligned}
A_{s} \sin \left(\alpha_{s} a\right)-\frac{M_{s}}{\alpha_{s}{ }^{2}} \cos \left(\alpha_{s} a\right)+\frac{m \beta^{2}}{\beta^{2}-\alpha_{s}{ }^{2}} \sin (\beta a) \\
+\frac{M_{s}}{\alpha_{s}{ }^{2}} \\
=A_{c} \sin \left(\alpha_{c} a\right)+B_{c} \cos \left(\alpha_{c} a\right)+\frac{m \beta^{2}}{\beta^{2}-\alpha_{c}{ }^{2}} \sin (\beta a) \\
+\frac{M_{c}}{\alpha_{c}{ }^{2}},
\end{aligned}
$$

while the elastic equilibrium at the bending spring, which can be expressed as

$$
P y_{s}(a)=K_{f}\left[y_{s}^{\prime}(a)-y_{c}^{\prime}(a)\right]+M_{r}
$$

leads to the last relation for the integration constants:

$$
\begin{gathered}
P\left[A_{s} \sin \left(\alpha_{s} a\right)-\frac{M_{s}}{\alpha_{s}{ }^{2}} \cos \left(\alpha_{s} a\right)+\frac{m \beta^{2}}{\beta^{2}-\alpha_{s}{ }^{2}} \sin (\beta a)\right. \\
\left.+\frac{M_{s}}{\alpha_{s}{ }^{2}}\right]=K\left[\alpha_{s} A_{s} \cos \left(\alpha_{s} a\right)+\alpha_{s} \frac{M_{s}}{\alpha_{s}{ }^{2}} \sin \left(\alpha_{s} a\right)\right. \\
\left.+\frac{m \beta^{3}}{\beta^{2}-\alpha_{s}{ }^{2}} \cos (\beta a)\right]-K\left[\alpha_{c} A_{c} \cos \left(\alpha_{c} a\right)\right. \\
\left.-\alpha_{c} B_{c} \sin \left(\alpha_{c} a\right)+\frac{m \beta^{3}}{\beta^{2}-\alpha_{c}{ }^{2}} \cos (\beta a)\right]+M_{r} .
\end{gathered}
$$

From relation (50), constant $B_{c}$ can be obtained as a function of $A_{c}$ :

$$
B_{c}=-A_{c} \tan \left[\alpha_{c}(a+b)\right]-\frac{M_{c}}{\alpha_{c}^{2} \cos \left[\alpha_{c}(a+b)\right]} .
$$


By substituting (54) in (51) and (53), the following system for the integration constants $A_{s}$ and $A_{c}$ is hence obtained:

$$
\begin{aligned}
& A_{s} \sin \left(\alpha_{s} a\right)-\frac{M_{s}}{\alpha_{s}^{2}} \cos \left(\alpha_{s} a\right)+\frac{m \beta^{2}}{\beta^{2}-\alpha_{s}^{2}} \sin (\beta a) \\
& +\frac{M_{s}}{\alpha_{s}{ }^{2}}=A_{c} \sin \left(\alpha_{c} a\right)-A_{c} \tan \left[\alpha_{c}(a+b)\right] \cos \left(\alpha_{c} a\right) \\
& -\frac{M_{c}}{\alpha_{c}{ }^{2} \cos \left[\alpha_{c}(a+b)\right]} \cos \left(\alpha_{c} a\right)+\frac{m \beta^{2}}{\beta^{2}-\alpha_{c}{ }^{2}} \sin (\beta a) \\
& +\frac{M_{c}}{\alpha_{c}^{2}} \\
& P\left[A_{s} \sin \left(\alpha_{s} a\right)-\frac{M_{s}}{\alpha_{s}{ }^{2}} \cos \left(\alpha_{s} a\right)+\frac{m \beta^{2}}{\beta^{2}-\alpha_{s}{ }^{2}} \sin (\beta a)\right. \\
& \left.+\frac{M_{s}}{\alpha_{s}^{2}}\right]=K\left[\alpha_{s} A_{s} \cos \left(\alpha_{s} a\right)+\alpha_{s} \frac{M_{s}}{\alpha_{s}^{2}} \sin \left(\alpha_{s} a\right)\right. \\
& \left.+\frac{m \beta^{3}}{\beta^{2}-\alpha_{s}^{2}} \cos (\beta a)\right]-K\left[\alpha_{c} A_{c} \cos \left(\alpha_{c} a\right)\right. \\
& +\alpha_{c} A_{c} \tan \left[\alpha_{c}(a+b)\right] \sin \left(\alpha_{c} a\right) \\
& +\frac{M_{c}}{\alpha_{c}^{2} \cos \left[\alpha_{c}(a+b)\right]} \alpha_{c} \sin \left(\alpha_{c} a\right) \\
& \left.+\frac{m \beta^{3}}{\beta^{2}-\alpha_{c}^{2}} \cos (\beta a)\right]+M_{r} \text {. }
\end{aligned}
$$

To obtain the solution for $A_{s}$ and $A_{c}$, the system can be rewritten in matrix form:

$$
\left[\begin{array}{ll}
C_{11} & C_{12} \\
C_{21} & C_{22}
\end{array}\right]\left\{\begin{array}{l}
A_{s} \\
A_{c}
\end{array}\right\}=\left\{\begin{array}{l}
T_{1} \\
T_{2}
\end{array}\right\}
$$

with

$$
\begin{aligned}
& C_{11}=\sin \left(\alpha_{s} a\right), \\
& C_{12}=\tan \left[\alpha_{c}(a+b)\right] \cos \left(\alpha_{c} a\right)-\sin \left(\alpha_{c} a\right), \\
& C_{21}=P \sin \left(\alpha_{s} a\right)-K \alpha_{s} \cos \left(\alpha_{s} a\right), \\
& C_{22} \\
& \quad=K\left\{\alpha_{c} \cos \left(\alpha_{c} a\right)+\alpha_{c} \tan \left[\alpha_{c}(a+b)\right] \sin \left(\alpha_{c} a\right)\right\},
\end{aligned}
$$

$T_{1}$

$$
\begin{aligned}
= & m \beta^{2} \sin (\beta a)\left(\frac{1}{\beta^{2}-\alpha_{c}^{2}}-\frac{1}{\beta^{2}-\alpha_{s}^{2}}\right) \\
& -\frac{M_{s}}{\alpha_{s}^{2}}\left[1-\cos \left(\alpha_{s} a\right)\right] \\
& +\frac{M_{c}}{\alpha_{c}^{2}}\left\{1-\frac{\cos \left(\alpha_{c} a\right)}{\cos \left[\alpha_{c}(a+b)\right]}\right\},
\end{aligned}
$$

$T_{2}$

$$
\begin{aligned}
= & K m \beta^{3} \cos (\beta a)\left(\frac{1}{\beta^{2}-\alpha_{s}^{2}}-\frac{1}{\beta^{2}-\alpha_{c}^{2}}\right) \\
& +P \frac{M_{s}}{\alpha_{s}^{2}} \cos \left(\alpha_{s} a\right)-P \frac{m \beta^{2}}{\beta^{2}-\alpha_{s}^{2}} \sin (\beta a)-P \frac{M_{s}}{\alpha_{s}^{2}} \\
& +K \alpha_{s} \frac{M_{s}}{\alpha_{s}^{2}} \sin \left(\alpha_{s} a\right) \\
& -\frac{K M_{c}}{\alpha_{c}^{2} \cos \left[\alpha_{c}(a+b)\right]} \alpha_{c} \sin \left(\alpha_{c} a\right)+M_{r} .
\end{aligned}
$$

The linear system can hence be solved according to Cramer's theorem for $A_{s}$ and $A_{c}$ :

$$
\begin{aligned}
& A_{s}=\frac{\left|\begin{array}{ll}
T_{1} & C_{12} \\
T_{2} & C_{22}
\end{array}\right|}{\left|\begin{array}{ll}
C_{11} & C_{12} \\
C_{21} & C_{22}
\end{array}\right|}=\frac{T_{1} C_{22}-T_{2} C_{12}}{C_{11} C_{22}-C_{12} C_{21}}, \\
& A_{c}=\frac{\left|\begin{array}{ll}
C_{11} & T_{1} \\
C_{21} & T_{2}
\end{array}\right|}{\left|\begin{array}{ll}
C_{11} & C_{12} \\
C_{21} & C_{22}
\end{array}\right|}=\frac{T_{2} C_{11}-T_{1} C_{21}}{C_{11} C_{22}-C_{12} C_{21}},
\end{aligned}
$$

while $B_{s}$ and $B_{c}$ can be deduced from (48) and (54), respectively. By imposing the determinant of system (56) to be zero, to find the limit load equation, the transcendental equation (43) is found again.

2.2. Experimental Setup. The model presented in Section 2.1.5 has been validated exploiting data from an experimental test campaign conducted in [10]. An actual realization of a hydraulic actuator was manufactured, according to the drawings reported in Figure 8(b), the overall layout being depicted as in Figure 8(a), showing the test layout presented in [10]. The actuator main dimensions are reported in Table 1 . The actuator was then linked, by exploiting threaded connections at the rod and cylinder ends, to removable pin junctions, which could mount ball-bearings supports.

As a first test, a varying pressure $p$ was applied to the cylinder, its value being measured along with the actual applied load $P$. The load was obtained by mounting an in-line load cell, with symmetric measurement behavior for tension and compression. The difference between the ideal load $P_{0}$, obtained from the applied pressure $p$ on the piston, and the measured load $P$ is reported in Figure 9. The actuator shows an efficiency $\eta_{p \max }=90.1 \%$ for an applied pressure of $156 \mathrm{MPa}$. The bending stresses are hence obtained by acquiring the signals of two couples of electrical linear strain gauges, positioned on the opposite sides of the rod as in Figure 8(a), and aligned with the axial direction of the rod. The SGs are placed at a distance $x_{\mathrm{sg}}=958 \mathrm{~mm}$ from the rod upper bearing. The bending stresses were reconstructed along two orthogonal planes as in Figure 8(a). The maximum bending stress $\sigma_{c}$ was measured by the two SGs placed on the orthogonal plane with respect to the ball-bearing supports, while the other two SGs confirmed a negligible bending on the parallel plane. 
TABLE 1: Dimensions and properties for the experimental actuator, from [10]—see Figures 4 and 8.

\begin{tabular}{lccccc}
\hline Parameter & Value & Parameter & Value & Parameter & Value \\
\hline$d_{s}[\mathrm{~mm}]$ & 20 & $f_{0}[\mathrm{~mm}]$ & 1.5 & $J_{s} \approx J_{p}\left[\mathrm{~mm}^{4}\right]$ & 7854 \\
$D_{\mathrm{ci}}[\mathrm{mm}]$ & 25 & $\delta_{1}[\mathrm{~mm}]$ & 27.1 & $J_{c}\left[\mathrm{~mm}^{4}\right]$ & 16286 \\
$D_{\mathrm{ce}}[\mathrm{mm}]$ & 30 & $\delta_{2}[\mathrm{~mm}]$ & 18.4 & $E_{s}, E_{c}[\mathrm{MPa}]$ & 206000 \\
$a[\mathrm{~mm}]$ & 1163 & $x_{p}[\mathrm{~mm}]$ & 63.6 & $E_{a}[\mathrm{MPa}]$ & 600 \\
$b[\mathrm{~mm}]$ & 1276 & $f$ & 0.07 & $K_{f}[\mathrm{Nmm} / \mathrm{mad}]$ & 26111000 \\
\hline
\end{tabular}

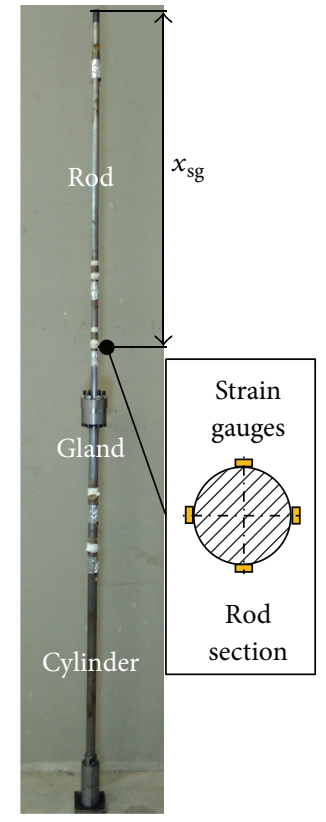

(a)

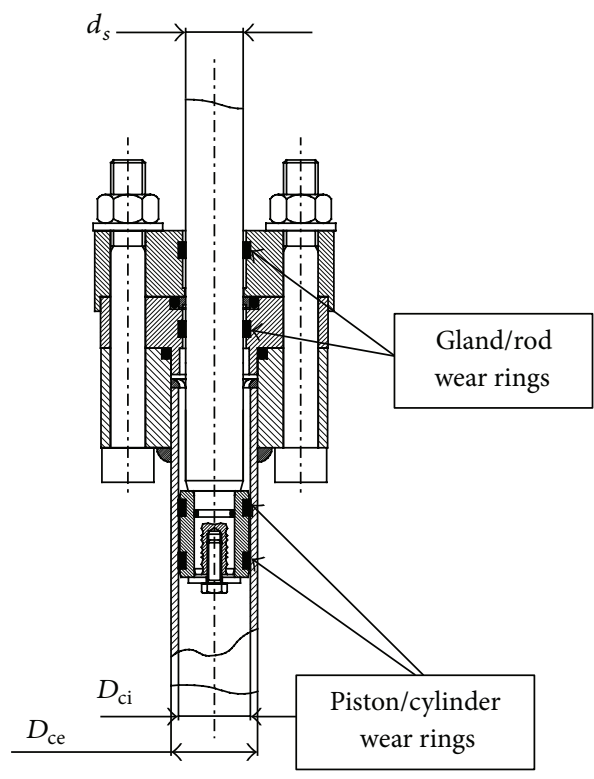

(b)

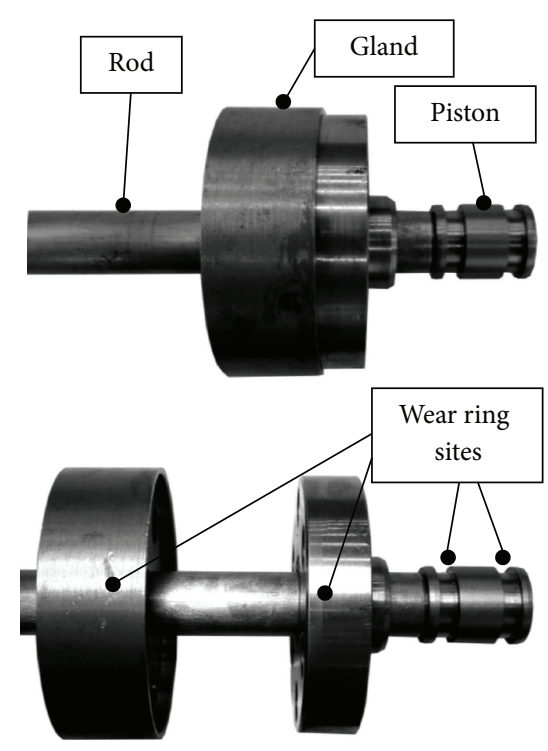

(c)

FIGURE 8: Realization of the actuator for the experimental tests performed in [10]: (a) picture of the actuator; (b) drawing of the rod/cylinder junction, with indication of wear rings positioning; (c) pictures of the gland, which could be opened for wear rings placement and substitution.

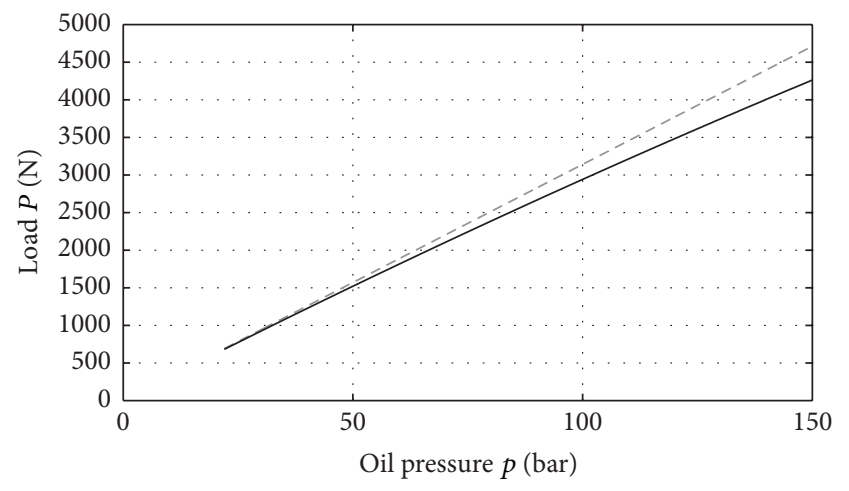

- Ideal compression load $F=p A$

- True compression load with $\eta_{p \max }=0.901$

Figure 9: Ideal and true compression load measured during experimental test.

2.3. Sensitivity Analysis. Based on the numerical model presented in Section 2.1, a sensitivity analysis has been performed on several geometrical and physical parameters.
The base model selected for the analysis is the combined model, presenting double wear rings on the piston-cylinder and on the gland-rod interfaces, friction moment, and initial rectilinear imperfections, as described in Section 2.1.5. The analyzed geometrical features are the influence of the distance between the piston and the gland wear rings $x_{p}$, as indicated in Figure 4, and the effect of dimensional changes on the wear rings thickness $s$ and width $t$. Such effects may be related to production tolerances and to loss of material due to wear on actuators subjected to long repetitions of work cycles. The material Young's modulus $E_{a}$ for the wear rings was also set as a parameter, since different possible commercial solutions were available for the material, according to $[9,10]$. For each combined geometrical and material variation on the wear rings, a linear FE model as presented in Section 2.1.4 was solved, obtaining the value of the linear stiffness $K$ as a function of these parameters.

Another parametric study regarded the friction coefficient $f$ on supports. In the present experimental tests, rolling bearings with limited friction moment contribution were adopted. In practical applications, however, steel pins without bearings may be used, or actual bearings may be 
TABLE 2: Parametrization for the sensitivity analysis, including default values and variation range.

\begin{tabular}{lcc}
\hline Parameter & Base value & Range \\
\hline Wear ring width $t[\mathrm{~mm}]$ & 6 & $5.5 \div 6$ \\
Wear ring thickness $s[\mathrm{~mm}]$ & 3 & $2.5 \div 3$ \\
Wear ring Young's modulus $[\mathrm{MPa}]$ & 600 & $260 \div 3000$ \\
Friction coefficient $f[-]$ & 0.07 & $0 \div 0.3$ \\
Distance $x_{p}$ between the wear rings $[\mathrm{mm}]$ & 63.6 & $20 \div 150$ \\
Rectilinear imperfection $f_{0}[\mathrm{~mm}]$ & 1.5 & $0 \div 3$ \\
\hline
\end{tabular}

worn or insufficiently lubricated. The Gamez-Montero et al. formulation [12] was adopted, in the form of (24), for different values of the friction coefficient. Table 2 reports the main parameters adopted for the sensitivity analysis, as well as the base parameters adopted for the combined model. The base parameters were chosen in agreement with the experimental test setup data [10]. Considering the range of the sensitivity analysis, wear ring Young's modulus was chosen according to the different materials present in commercial wear rings [9, 10], while the dimensions vary from the standard base value considering tolerance imprecisions and material reduction due to wear. Friction coefficient has been varied between very lubricated and very dry steel-on-steel conditions, whereas the distance $x_{p}$ is a choice of the designer. The rectilinear imperfection has been varied from zero to twice the imperfection found on the experimental realization.

\section{Results and Discussion}

3.1. Numerical Model Validation. A validation of the numerical model was performed, based on the experimental data obtained according to the test described in Section 2.2. The parameters settings, matching the actual conditions of the test configuration, have been reported in Table 1. The test settings are indeed corresponding to the base values adopted for the numerical model in the sensitivity analysis. The results are reported in Figure 10, where the solid line represents the model with bending stiffness calculated considering the wear rings mounted both on the piston and on the gland, as in Figure 4 and (37). This configuration is matching with the actual experimental condition, in which two wear rings were mounted on the piston, and other two between the gland and the rod. To ensure consistency with the test conditions, the SKF model has been adopted for the friction moment, according to (25), with a ball-bearing diameter $d_{\mathrm{bb}}$ of $30 \mathrm{~mm}$.

The outputs from Timoshenko's model, without bending stiffness, and from the model considering wear rings between gland and rod alone-(33) - are reported with dashed and dotted lines, respectively. From Figure 10, it can be seen that, whilst the full model with hyperstatic determination of bending stiffness adequately reproduces the testing conditions, Timoshenko's model overestimates the limit load, underestimating the applied stresses due to bending under compression loads. The $K_{f}$ calculated with the wear rings on the gland/rod interaction gives reduced limit load and higher applied stresses at lower load values, indicating a reduced stiffness with respect to the full model calculated according
TABLE 3: Limit load and bending stiffness results according to different models.

\begin{tabular}{lcc}
\hline Model & Limit load $[\mathrm{N}]$ & $K_{f}[\mathrm{~N} \mathrm{~mm} / \mathrm{rad}]$ \\
\hline Timoshenko's, no $K_{f}$ & 3855 & - \\
2 wear rings, rod only & 3111 & $8071 \cdot 10^{3}$ \\
4 wear rings, rod and piston & 3629 & $29777 \cdot 10^{3}$ \\
\hline
\end{tabular}

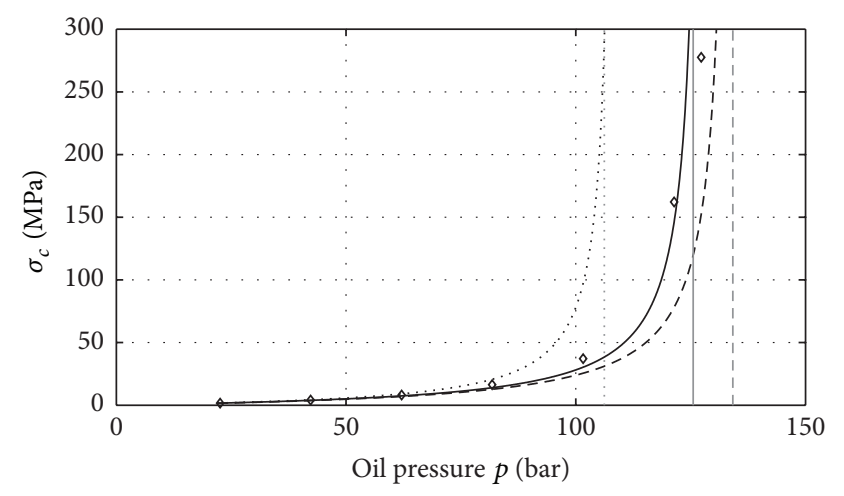

• Exp. data from [10] - pin junction, wear rings on gland and piston

- - - Pure Timoshenko model, no $K_{f}$

- $K_{f}$ hyperstatic model-wear rings on gland and piston

..... $K_{f}$ base model-wear rings on piston only

- Limit load-pure Timoshenko model, no $K_{f}$-equation (11)

_ Limit load $-K_{f}$ hyperstatic model-wear rings on gland and piston-equation (43)

Limit load $-K_{f}$ base model-wear rings on piston onlyequation (43)

FIgURE 10: Validation of the actuator numerical model against experimental data. Continue line: wear rings on the piston and on the gland; dotted line: wear rings on the gland only; dashed line: Timoshenko model with no $K_{f}$.

to (37). The limit loads, obtained from (43) for the models with bending stiffness, and from (11) for Timoshenko's model, are reported in Table 3, along with the bending stiffness values from the double wear rings or quadruple wear rings configurations. The bending stiffness has been obtained by considering a linear stiffness $K$ value of 11802 [N/mm], according to the $\mathrm{FE}$ solution for wear ring properties $E=$ $600 \mathrm{MPa}$ and $v=0.3$. The wear rings properties were chosen according to the wear ring type actually mounted during the experimental test $[9,10]$.

Starting from the numerical models presented in the previous section, the 4 wear rings model has been chosen as the reference model for the sensitivity analysis. The hyperstatic formulation of the bending stiffness $K_{f}$, considering four wear rings, two mounted between the rod and the gland and other two mounted at the piston/cylinder interface, has indeed showed the best consistence with experimental results.

\subsection{Sensitivity Analysis Results}

3.2.1. Friction on the Supports. The friction moment on the pin support has been modeled according to the formulation provided by Gamez-Montero et al. in [12], as expressed 


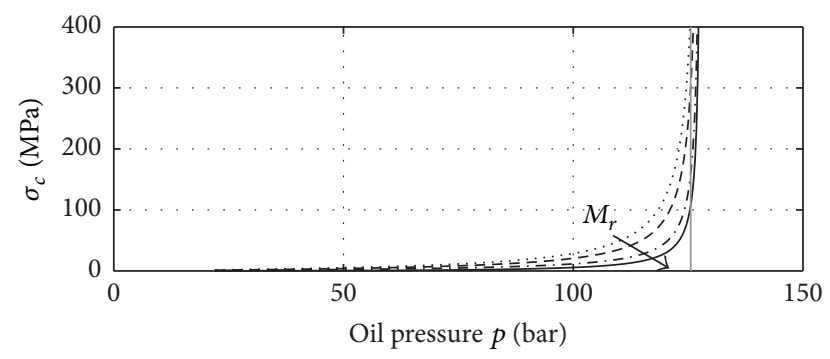

$$
\begin{array}{ll}
\ldots . . f & =0 \\
---f & =0.07 \\
--f & =0.15
\end{array} \quad-\quad \begin{aligned}
& f=0.30 \\
& \text { Limit load-equation (43) }
\end{aligned}
$$

Figure 11: Effect of the friction coefficient $f$ on the pin supports versus the bending stress distribution.

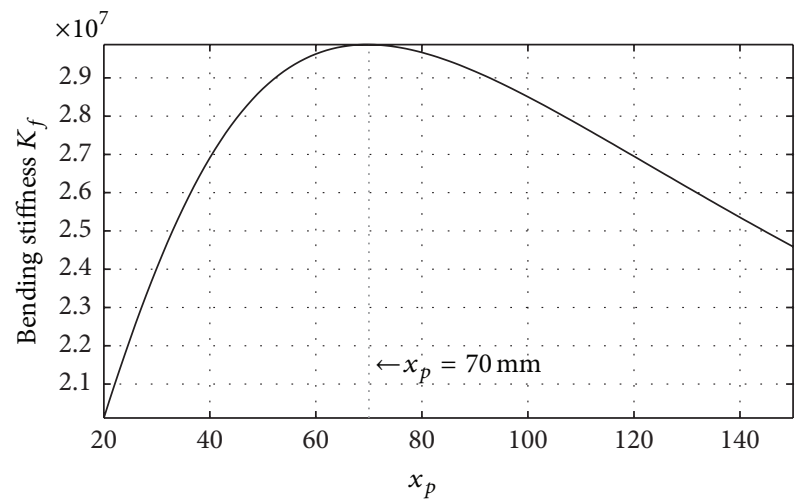

FIGURE 12: Bending stiffness for $x_{p}=70 \mathrm{~mm}$ behavior, function of the variation of the distance between rod and piston wear rings $x_{p}$.

in (24). The results are displayed in Figure 11. It can be noted that the limit value seems to be not affected by the amount of friction, according to (43). Indeed, friction appears only between the known terms in the solving system (56). However, lower amounts of friction produce a steeper approach, in terms of bending stress, to the limit load. The applied stresses are hence lower for real pin supports where high coefficients of friction are supposed to exist. This result indicates that the maximum bending load is dependent on the actual constraint conditions, which have to be assessed for operating actuators.

3.2.2. Spacing between Wear Rings. A construction detail, which may affect the bending behavior of the actuator, is defined by the actual distance between the wear rings mounted on the piston and on the rod; see $x_{p}$ in Figure 4. The base value of such distance was, in the actual realization of the device tested in Section 2.2, of $63.6 \mathrm{~mm}$, considering a full extension of the rod. By modifying $x_{p}$, a sensible effect on the bending stiffness is obtained, as reported in Figure 12. Particularly, $K_{f}$ shows a maximum value for $x_{p}=70 \mathrm{~mm}$.

The stress pattern displayed in Figure 13, which is obtained accordingly with the $x_{p}$ variation, shows an increased limit load for $x_{p}=70 \mathrm{~mm}$. This effect is beneficial,

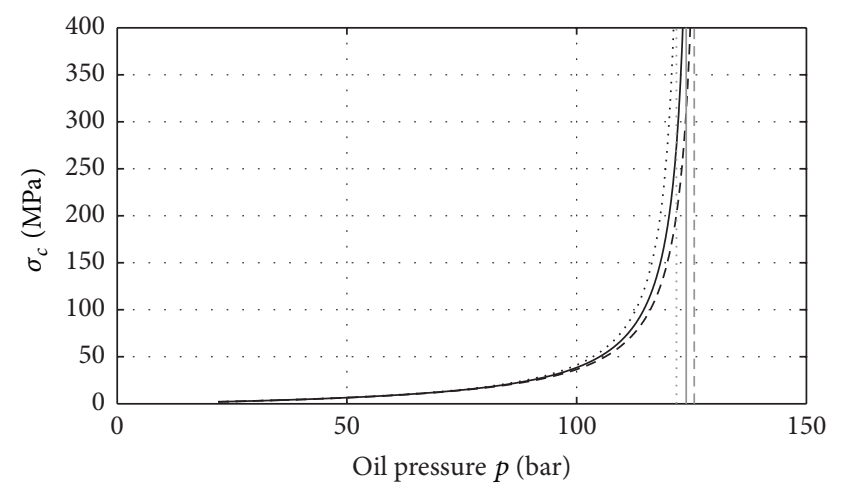

$$
\begin{aligned}
& \text { … } x_{p}=20 \mathrm{~mm} \quad \ldots \text { Limit load }-x_{p}=20 \mathrm{~mm} \text {-equation (43) } \\
& --x_{p}=70 \mathrm{~mm} \quad \ldots \text { Limit load }-x_{p}=70 \mathrm{~mm} \text {-equation (43) } \\
& -x_{p}=150 \mathrm{~mm} \longrightarrow \text { Limit load }-x_{p}=150 \mathrm{~mm} \text {-equation (43) }
\end{aligned}
$$

FIGURE 13: Bending stress behavior according to the modification of the wear rings distance $x_{p}$.

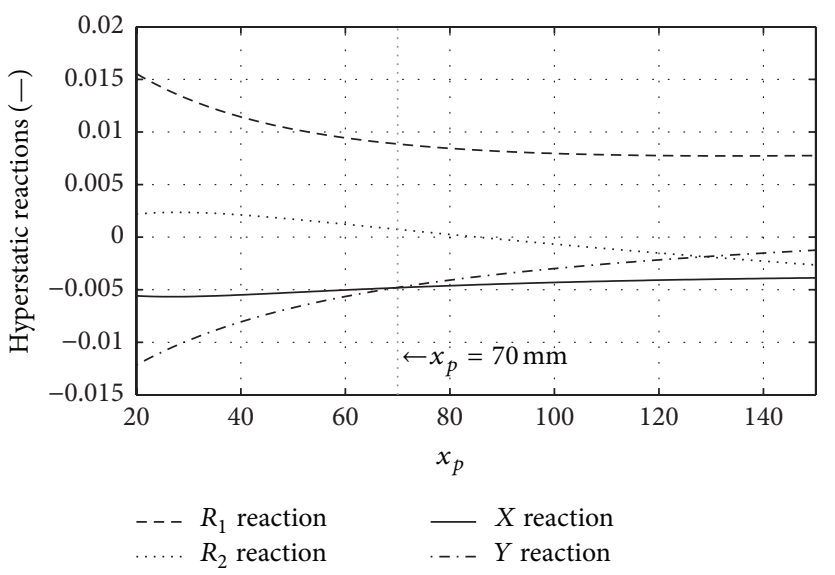

FIGURE 14: Hyperstatic reactions behavior versus the wear rings distance $x_{p}$.

since it decreases the maximum stress along the actuator for the same applied load.

In order to assess the causes underlying this behavior, the values of the reactions of the virtual system used for the determination of $K_{f}$ are displayed in Figure 14, versus the value of $x_{p}$. Hyperstatic unknown reactions $X$ and $Y$ are obtained by solving the PVW system (34). The other two reaction forces, indicated as $H_{1}$ and $H_{2}$, as in Figure 4, are obtained by rotational equilibrium:

$$
\begin{aligned}
& H_{1}=\frac{1}{\delta_{1}}\left[C+x_{p} X+\left(x_{p}+\delta_{2}\right) Y\right] \\
& H_{2}=-\frac{1}{\delta_{1}}\left[C+\left(x_{p}+\delta_{1}\right) X+\left(x_{p}+\delta_{1}+\delta_{2}\right) Y\right] .
\end{aligned}
$$

From Figure 14, it can be noticed that, for $x_{p}<70 \mathrm{~mm}$, the most external reactions, namely, $H_{1}$ and $Y$, grow significantly. This fact indicates that, if the distance $x_{p}$ is shortened below $70 \mathrm{~mm}$, the springs positioned at the extremes experience an increased elongation, in order to counteract the applied 


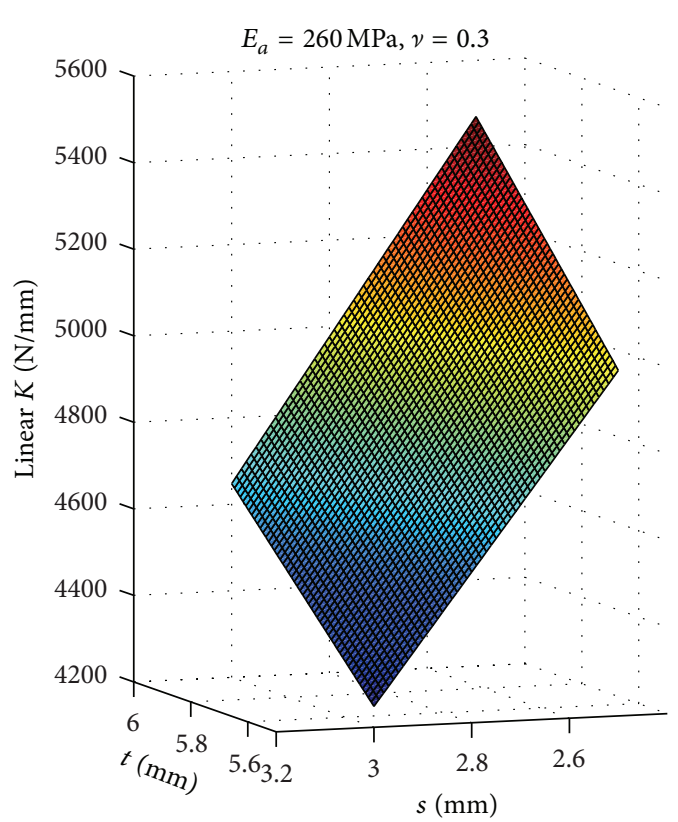

(a)

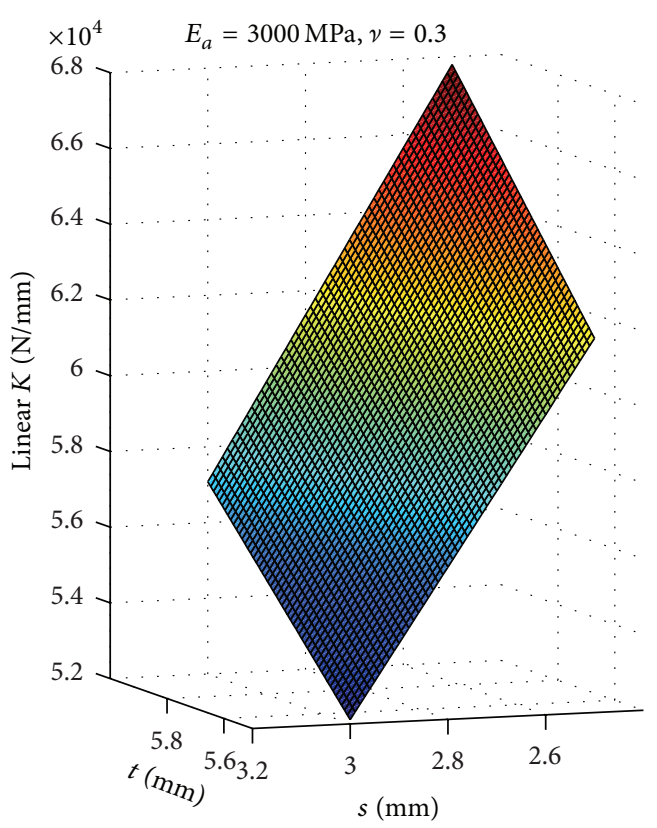

(b)

FIGURE 15: Wear rings linear stiffness $K$ dependence over width $t$ and thickness $s$ : (a) wear rings modulus $E_{a}=260 \mathrm{MPa}$; (b) wear rings modulus $E_{a}=3000 \mathrm{MPa}$.

moment $C$. The rotation required to counteract the moment is higher in this case, thus leading to a decrease of the bending stiffness $K_{f}$, as reported in Figure 12. For values of $x_{p}$ higher than $70 \mathrm{~mm}$, on the other hand, the reaction forces tend to stable values. The decrease in $K_{f}$ is in this latter case owed to the flexibility of the rod between the gland and the piston. Indeed, the free length of the rod between the gland and the piston increases with $x_{p}$, producing nonnegligible contributions for high values. The balance between these two trends identifies an optimum value for $x_{p}$, which for the present geometry and adopted materials is equal to $70 \mathrm{~mm}$. This result indicates that $x_{p}$ can be optimized, in the design of actuators, to obtain higher limit loads and reduced bending stresses at higher applied pressures.

\subsubsection{Wear Rings Material and Geometry. As already} reported in Section 2.3, different wear rings with different materials can be used to equip a hydraulic actuator $[9,10]$. Besides, geometrical tolerances and wear can modify the actual ring dimensions. In order to take into account these effects on the linear stiffness $K$ of the wear rings, several FE models have been made, according to the procedure reported in Section 2.1.4. As reported in Table 2, the parameters for this analysis were the wear ring thickness $t$ and width $s$, as well as the actuator material, identified with its Young's modulus $E_{a}[9,10]$. From Figure 15, the contribution of the geometrical dimensions of the wear rings on the linear stiffness $K$ can be pointed out. The wear rings present an increased stiffness for high $s / t$ ratios, meaning that thin wear rings with a large base increase the linear rigidity of the system.

The influence of the material modulus $E_{a}$ is reported in Figure 16, for different geometrical configurations.

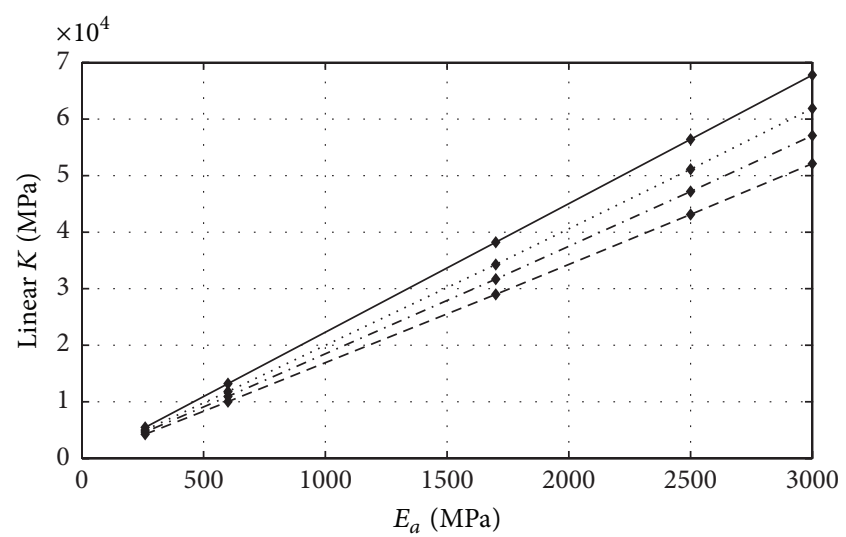

$\begin{aligned} \rightarrow-\text { Width } t & =5.5 \mathrm{~mm} \text {, thickness } s=2.5 \mathrm{~mm} \\ \longrightarrow-\text { Width } t & =6 \mathrm{~mm} \text {, thickness } s=2.5 \mathrm{~mm} \\ \rightarrow-\text { Width } t & =5.5 \mathrm{~mm} \text {, thickness } s=3 \mathrm{~mm} \\ \rightarrow-\text { Width } t & =6 \mathrm{~mm} \text {, thickness } s=3 \mathrm{~mm}\end{aligned}$

FIGURE 16: Wear rings linear stiffness $K$ dependence with wear rings Young's modulus $E_{a}$.

The relation between $E_{a}$ and $K$ is linear, as expected from a linear representation of the material in the FE model, its slope related to the geometrical dimensions of the ring.

Although the relationship between $E_{a}$ and $K$ is linear, the contribution of the wear ring material on the system behavior is more complex. By examining Figure 17, it is found that the relationship between the chosen wear rings material, that is, $E_{a}$, and the bending stiffness of the rod/cylinder interface is not linear. The nonlinearity of the behavior is indeed caused by the hyperstatic system formed by the wear rings. 


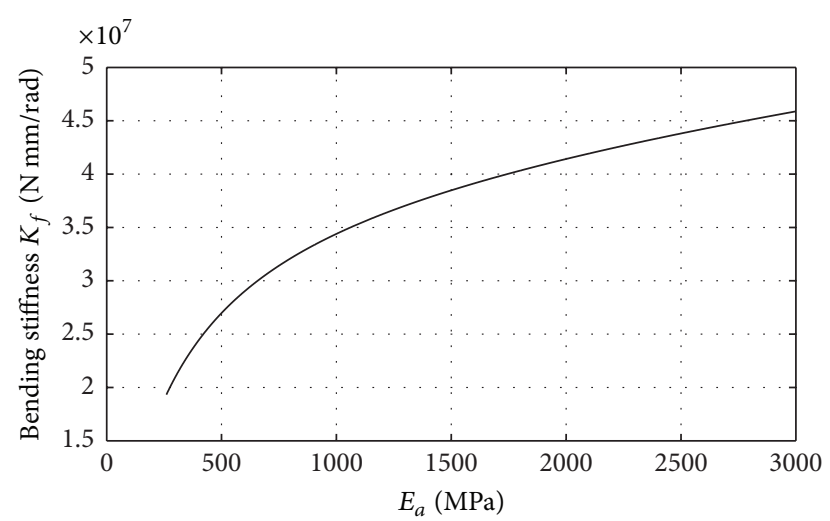

FIGURE 17: Bending stiffness $K_{f}$ dependence with wear rings Young's modulus $E_{a}$.

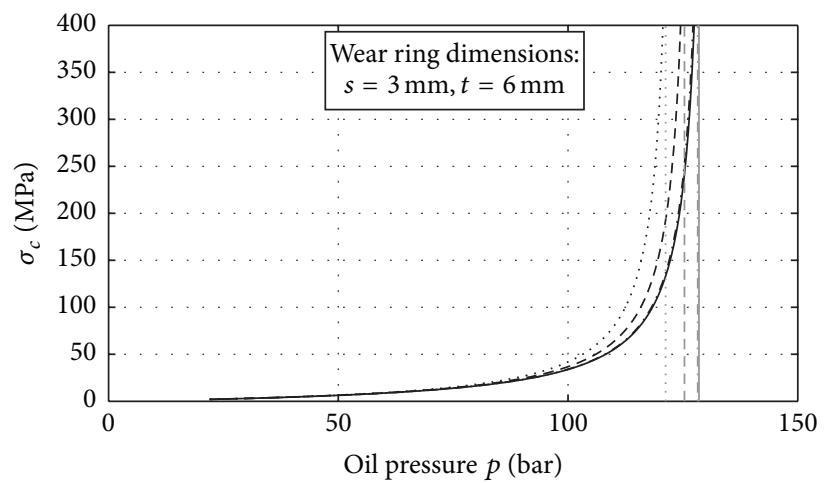

… $E_{a}=260 \mathrm{MPa}$

-- $E_{a}=600 \mathrm{MPa}$

-.. $E_{a}=2500 \mathrm{MPa}$

- $E_{a}=3000 \mathrm{MPa}$

$$
\begin{aligned}
& \text { … Limit load- } E_{a}=260 \mathrm{MPa}-\text { equation (43) } \\
& \text {-- Limit load- } E_{a}=600 \mathrm{MPa}-\text { equation (43) } \\
& \text {-.- Limit load- } E_{a}=2500 \mathrm{MPa}-\text { equation (43) } \\
& -E_{a}=3000 \mathrm{MPa}-\text { equation (43) }
\end{aligned}
$$

FIGURE 18: Bending stress behavior according to the modification of the wear rings elastic modulus $E_{a}$.

The consequence of this nonlinear behavior is reflected in the choice of the wear ring material. Indeed, at lower values of $E_{a}$, that is, $260 \div 600 \mathrm{MPa}$, a variation on the material Young modulus affects significantly the bending behavior on the actuator. Wear rings with superior stiffness behavior, that is, $E_{a}=2500 \div 3000 \mathrm{MPa}$, are less affected by tolerances on the material quality. Indeed, in Figure 18, it can be seen that the bending stress curves and limiting loads for $E_{a}=2500 \mathrm{MPa}$ and $E_{a}=3000 \mathrm{MPa}$ are almost coincident.

3.2.4. Initial Deflection due to Rectilinear Imperfections. To conclude the sensitivity analysis, a determination of the contribution of the initial deflection caused by geometrical clearances has been performed. The model adopted for the analysis presented an initial transversal maximum displacement $f_{0}=1.6 \mathrm{~mm}$, as measured from the experimental setup. The results, considering different values for the initial displacement, are reported in Figure 19.

From Figure 19, it can be seen that a low initial displacement presents indeed reduced bending stresses up to very high values of applied pressure. The limit load seems to be not affected from the value of $f_{0}$, since the terms affected by the initial displacement are not present in (43). For zero displacement, the value of bending stress seems to be low when approaching the limit load value, as shown in Figure 19(a). Moreover, the numerical solution presents an instability at a value of applied pressure slightly lower with respect to the limit predicted by (43). This aspect suggests that the forcing terms contained in system (56) are slightly affecting the limit load too. Indeed, dependence on the applied compression load $P$ is found in the forcing terms $T_{1}$ and $T_{2}$ (61), (62) of system (56). However, the definition of an equation for the limit load $P$ which takes into account such contributions would take a significant analytical effort. The limit load found by (43) can be hence used, since it gives a good esteem also without the contribution of the forcing terms. Besides this fact, the behavior of the numerical model in the proximity of the limit load is also conditioned by friction, which appears as well in the forcing terms $T_{1}$ and $T_{2}$ of system (56). This aspect is highlighted in the comparison between Figures 19(a) and 19(b) presenting bending stresses at different initial displacement $f_{0}$ values for friction coefficients at the pin of $f=0.07$ and $f=0.30$, respectively. For low friction coefficients, that is, Figure 19(a), the bending stresses rise well before the limit load instability, and the vertical asymptote is moved on the left, if compared to the numerical limit load calculated from (43). Opposite considerations are made for high friction coefficients, as in Figure 19(b). From these results, it can be deduced that higher friction at the pin supports leads to reduce bending stresses, especially for very low initial rectilinear imperfection. Low stresses can be maintained up to the limit load, while on the other hand they will rise very steeply approaching the instability value.

\section{Conclusions}

In the present work, a complete theoretical model has been assembled, joining contributions from the most advanced descriptions found in the literature. The theoretical model accounted for the different inertial properties of the rod and of the cylinder. The contribution of the actual joint realization by means of wear rings has been modeled inserting the bending stiffness $K_{f}$ in the model. The effects of initial rectilinear imperfections due to geometrical clearances and of friction moments have been included. A numerical model, based on such theoretical premises, has been developed and validated against experimental testing. The numerical model has been then used to perform a sensitivity analysis over several parameters affecting the design and the operational conditions of the actuator. The sensitivity analysis pointed out the following key aspects related to the bending behavior of hydraulic actuators under compression:

(i) The friction on the supports does not alter significantly the limit load of the actuator. However, lower values of $f$, resulting in lower friction moments, increase the bending stress contribution, especially at higher loads.

(ii) A similar behavior is found with respect to initial displacements, that is, rectilinear imperfections. Low 


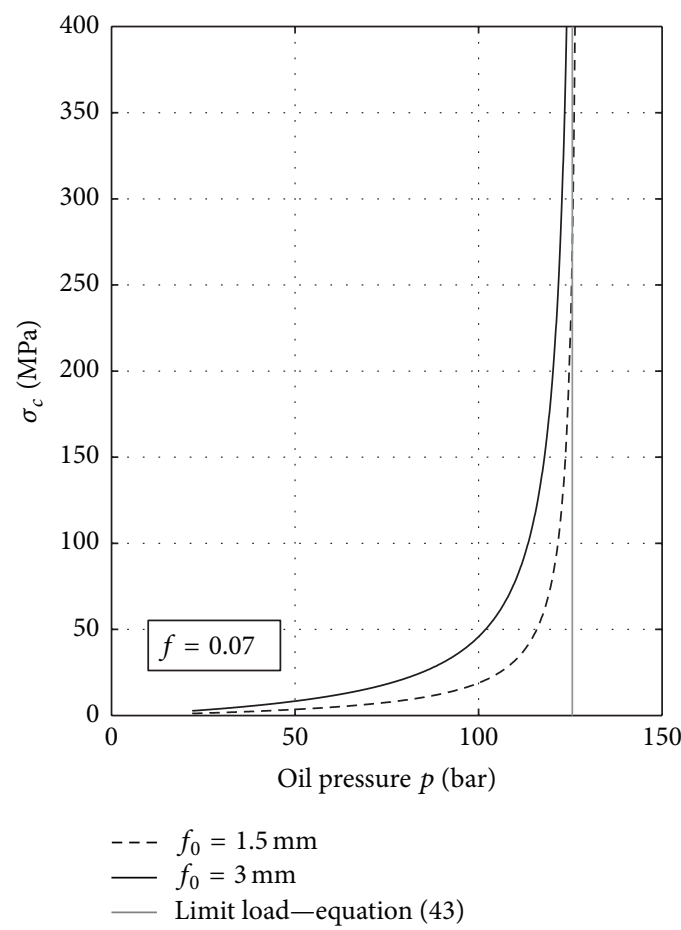

(a)

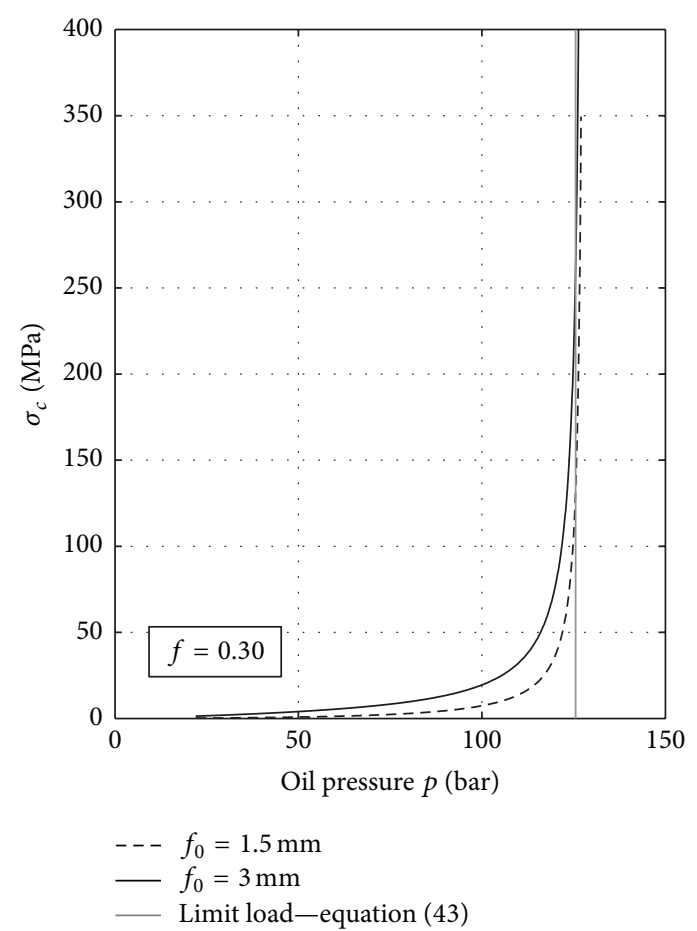

(b)

FIGURE 19: Bending stress behavior according to the initial maximum displacement $f_{0}$ : (a) friction coefficient $f=0.07$; (b) friction coefficient $f=0.30$.

initial displacements reduce significantly the amount of bending stresses for high values of applied pressure, near the limit load.

(iii) The combined influence of friction and rectilinear imperfection notably influences the values of bending stress for hydraulic actuators at high applied loads. Significant levels of stresses are reached on actuators subjected to low friction and notable initial deflections, for values of applied load inferior with respect to the instability load. This behavior may lead to critical situations in actual operating conditions.

(iv) The distance $x_{p}$ between the wear rings mounted on the piston and on the gland influences the bending stiffness, and hence the limit load of the actuator. An optimum value can be found to maximize the limit load, as a compromise between high deflections caused by the high hyperstatic reactions at low $x_{p}$ values, and the nonnegligible bending of the rod at high $x_{p}$ values.

(v) The wear rings material and size affect the bending stiffness of the actuator, and hence the limit value. Wear rings with high $E_{a}$ values increase the $K_{f}$ value, thus leading to increased limit loads. The bending stress behavior near the threshold load value is affected as well. The sensitivity of $K_{f}$ to the wear rings Young's modulus is reduced for high $E_{a}$ values.

According to the conclusions found, the present model is a useful tool for the designer, accounting properly for the effects of geometrical imperfections and friction moments on the joints on the bending stresses on the actuator. The model gives also information about the choice of the appropriate material for the wear rings, in order to reduce the limit load. Moreover, the numerical model helps in the correct dimensioning of the distance between the wear rings on the gland and on the rod, in order to maximize the limit load for a given extension of the actuator. Further experimental work will help to confirm the results obtained from the sensitivity model. However, the results from the numerical analysis highlighted that the effects of geometrical clearances, wear rings, and friction on the supports are not negligible in the design of hydraulic actuators subjected to high applied loads.

\section{Nomenclature}

$\alpha$ [rad]: Circumferential wear rings reference direction

$\delta[\mathrm{mm}]: \quad$ Distance between wear rings on the gland, 2 wear rings model

$\delta_{1}[\mathrm{~mm}]:$ Distance between wear rings on the gland, 4 wear rings model

$\delta_{2}[\mathrm{~mm}]:$ Distance between wear rings on the piston, 4 wear rings model

$\Delta[\mathrm{mm}]: \quad$ Deformed wear ring thickness FEM

$\Delta_{0}[\mathrm{~mm}]:$ Initial wear ring thickness FEM

$\Delta x[\mathrm{~mm}]:$ Relative displacement of the wear ring thickness FEM

$\Delta s_{m}[\mathrm{~mm}]:$ Mean wear ring displacement 


\begin{tabular}{|c|c|}
\hline$\theta$ [rad]: & $\begin{array}{l}\text { Rotation angle between rod and } \\
\text { cylinder }\end{array}$ \\
\hline$a[\mathrm{~mm}]:$ & Extended rod length \\
\hline$b[\mathrm{~mm}]:$ & Cylinder length \\
\hline$C[1 \cdot \mathrm{mm}]:$ & Virtual couple applied in PVW \\
\hline$d_{s}[\mathrm{~mm}]:$ & Rod shaft diameter \\
\hline$D_{\text {ci }}[\mathrm{mm}]:$ & Cylinder internal diameter \\
\hline$D_{\text {ce }}[\mathrm{mm}]:$ & Cylinder external diameter \\
\hline$d_{\text {pin }}[\mathrm{mm}]:$ & Diameter of the pin support \\
\hline$d_{\mathrm{bb}}[\mathrm{mm}]:$ & Diameter of the ball-bearing support \\
\hline$d F[\mathrm{~N}]:$ & $\begin{array}{l}\text { Elementary force along the wear ring } \\
\text { circumferential direction }\end{array}$ \\
\hline$d M[\mathrm{~N} \mathrm{~mm}]:$ & Elementary moment generated by $d F$ \\
\hline$E_{a}[\mathrm{MPa}]:$ & Wear rings Young's modulus \\
\hline$E_{s}[\mathrm{MPa}]:$ & Young's modulus of the rod shaft \\
\hline$E_{c}[\mathrm{MPa}]:$ & Cylinder Young's modulus \\
\hline$f[-]:$ & Pin friction coefficient \\
\hline$f_{\mathrm{bb}}[-]:$ & Ball-bearing friction coefficient \\
\hline$f_{0}[\mathrm{~mm}]:$ & Maximum transversal displacement \\
\hline$H_{1}, H_{2}, X, Y[-]:$ & Virtual reactions from PVW \\
\hline$J_{s}\left[\mathrm{~mm}^{4}\right]:$ & Section inertia of the shaft rod \\
\hline$J_{p}\left[\mathrm{~mm}^{4}\right]:$ & Section inertia of the piston \\
\hline$J_{c}^{P}\left[\mathrm{~mm}^{4}\right]:$ & Section inertia of the cylinder \\
\hline$K, K_{1}, K_{2}[\mathrm{~N} / \mathrm{mm}]:$ & Linear stiffness of the wear rings \\
\hline$K_{f}[\mathrm{~N} \mathrm{~mm} / \mathrm{rad}]:$ & Bending stiffness of the system \\
\hline$l[\mathrm{~mm}]:$ & Unsupported length of column \\
\hline$M_{r}[\mathrm{Nmm}]:$ & Friction moment at the pin ends \\
\hline$P[\mathrm{~N}]:$ & Applied compression load \\
\hline$P_{0}[\mathrm{~N}]:$ & Ideal compression load \\
\hline$p$ [bar]: & Internal cylinder pressure \\
\hline$p_{m}[\mathrm{MPa}]:$ & $\begin{array}{l}\text { Maximum pressure exerted on the } \\
\text { wear ring }\end{array}$ \\
\hline$s[\mathrm{~mm}]:$ & Wear rings thickness \\
\hline$t[\mathrm{~mm}]:$ & Wear rings width \\
\hline$x[\mathrm{~mm}]:$ & Axial coordinate along the actuator \\
\hline$x_{p}[\mathrm{~mm}]:$ & $\begin{array}{l}\text { Distance between the nearest wear } \\
\text { rings on the gland and on the piston, } 4 \\
\text { wear rings model }\end{array}$ \\
\hline$x_{\mathrm{sg}}[\mathrm{mm}]:$ & $\begin{array}{l}\text { Distance of the strain gauges } \\
\text { measurement point }\end{array}$ \\
\hline$y_{s}[\mathrm{~mm}]:$ & $\begin{array}{l}\text { Transversal displacement of the rod } \\
\text { shaft }\end{array}$ \\
\hline$y_{c}[\mathrm{~mm}]:$ & $\begin{array}{l}\text { Transversal displacement of the } \\
\text { cylinder }\end{array}$ \\
\hline$y_{0}[\mathrm{~mm}]:$ & Initial displacement distribution. \\
\hline
\end{tabular}

\section{Conflict of Interests}

The authors declare that no conflict of interests exists regarding the present paper.

\section{References}

[1] S. Smiles, Industrial Biography: Iron-Workers and Tool-Makers, Ticknor and Fields, Boston, Mass, USA, 1864.

[2] B. Spear, "Joseph Brahmah-engineer, inventor and prolific patentee," World Patent Information, vol. 40, pp. 51-53, 2015.
[3] F. Bleich, Buckling Strength of Metal Structures, McGraw-Hill, New York, NY, USA, 1952.

[4] O. Belluzzi, Scienza delle Costruzioni, vol. 4, Zanichelli, Bologna, Italy, 1961.

[5] S. P. Timoshenko and J. M. Gere, Theory of Elastic Stability, McGraw-Hill, Tokyo, Japan, 1961.

[6] W. Flügge, Stresses in Shells, Springer, Berlin, Germany, 2nd edition, 1973.

[7] F. Hoblit, "Critical buckling for hydraulic actuating cylinders," in Stress Engineering, Product Engineering, pp. 108-112, Lockheed Aircraft Corporation, Burbank, Calif, USA, 1950.

[8] K. Seshasai, W. Dawkins, and S. Iyengar, "Stress analysis of hydraulic cylinders," in Proceedings of the National Conference on Fluid Power, Oklahoma State University, 1975.

[9] S. Baragetti and A. Terranova, "Limit load evaluation of hydraulic actuators," International Journal of Materials and Product Technology, vol. 14, no. 1, pp. 50-73, 1999.

[10] S. Baragetti and A. Terranova, "Bending behavior of doubleacting hydraulic actuators," Proceedings of the Institution of Mechanical Engineers, Part C: Journal of Mechanical Engineering Science, vol. 215, no. 5, pp. 607-619, 2001.

[11] P. J. Gamez-Montero, E. Salazar, R. Castilla, J. Freire, M. Khamashta, and E. Codina, "Misalignment effects on the load capacity of a hydraulic cylinder," International Journal of Mechanical Sciences, vol. 51, no. 2, pp. 105-113, 2009.

[12] P. J. Gamez-Montero, E. Salazar, R. Castilla, J. Freire, M. Khamashta, and E. Codina, "Friction effects on the load capacity of a column and a hydraulic cylinder," International Journal of Mechanical Sciences, vol. 51, no. 2, pp. 145-151, 2009.

[13] J. P. Lurot, "Hydraulic fluid power-cylinders: method for determining the buckling load," Tech. Rep. ISO/TS 13725, ISO Standard, 2001.

[14] X. Chen, F. Chen, J. Zhou, L. Li, and Y. Zhang, "Cushioning structure optimization of excavator arm cylinder," Automation in Construction, vol. 53, pp. 120-130, 2015.

[15] SKF Ball Bearings Online Catalog_Estimating the frictional moment, December 2015, http://www.skf.com/group/products/ bearings-units-housings/ball-bearings/principles/friction/estimating-frictional-moment/index.html. 


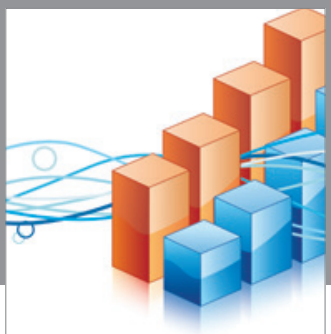

Advances in

Operations Research

vatem alat4

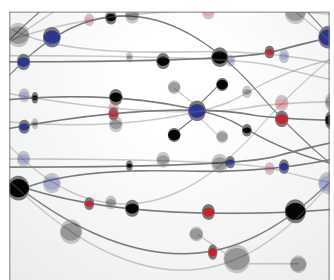

\section{The Scientific} World Journal
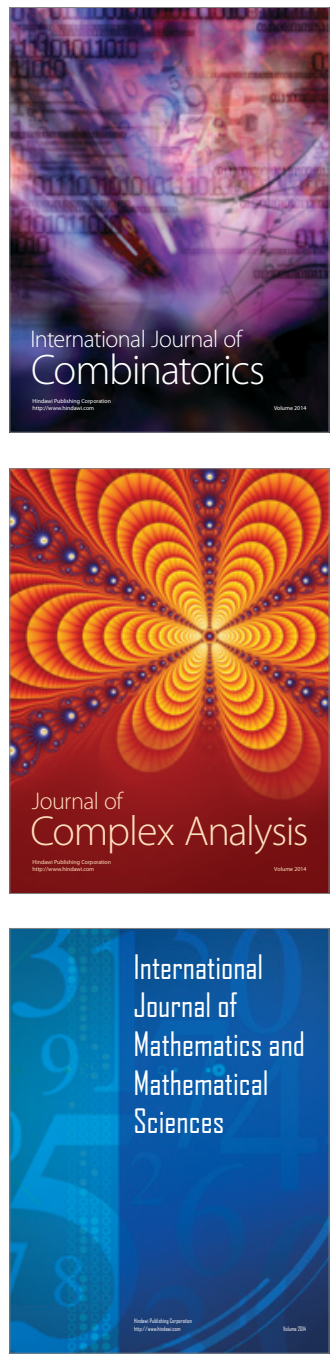
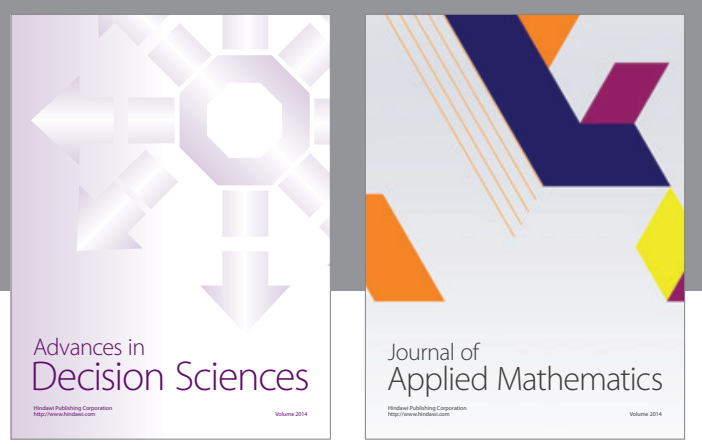

Algebra

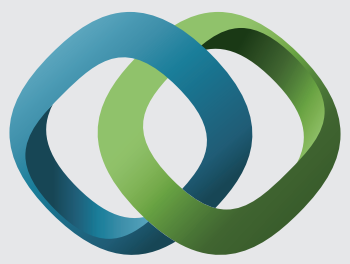

\section{Hindawi}

Submit your manuscripts at

http://www.hindawi.com
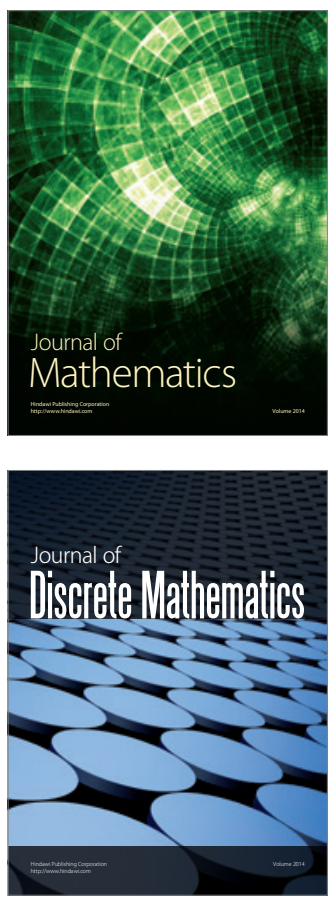

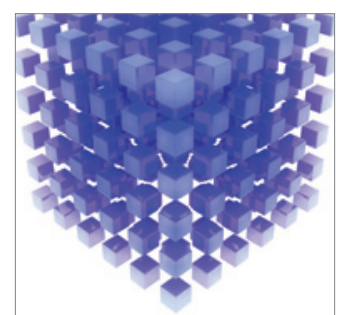

Mathematical Problems in Engineering
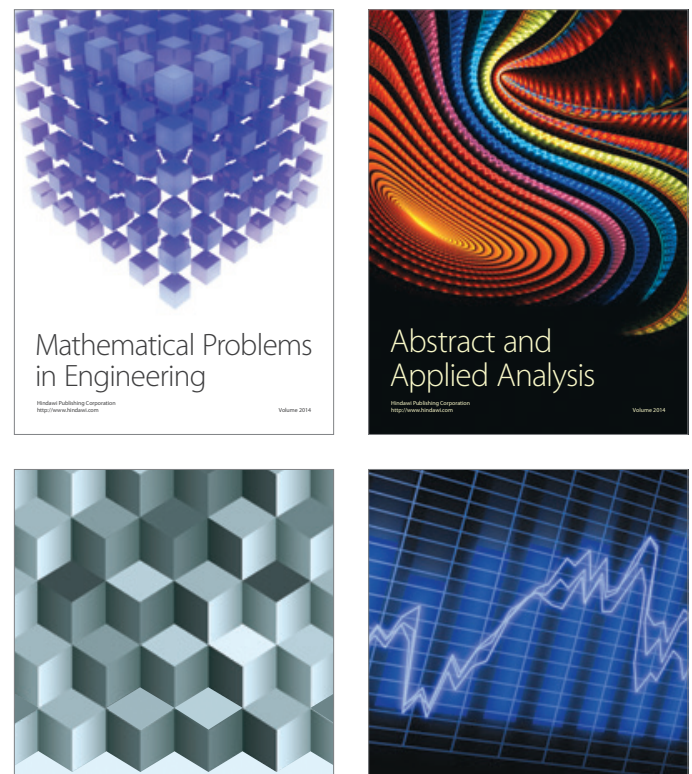

Journal of

Function Spaces

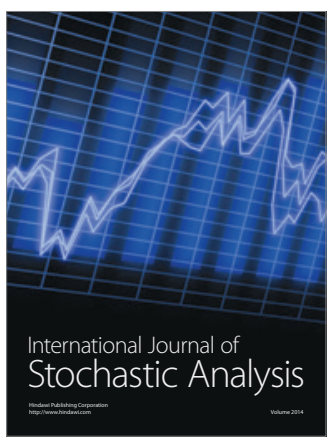

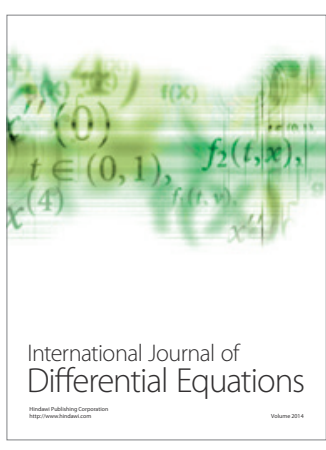
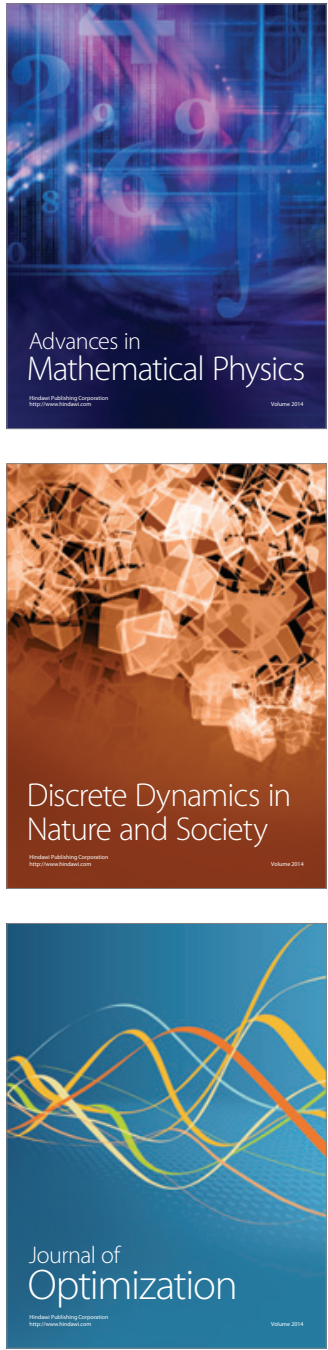